\title{
Nonadiabatic electron gun at an electron beam ion source: Commissioning results and charge breeding investigations
}

\author{
H. Pahlø, ${ }^{*}$ N. Bidault $\odot,{ }^{\dagger}$ G. Khatri®, A. Pikin $\oplus^{\ddagger}$ and F. J. C. Wenander $\odot^{\S}$ \\ European Organization for Nuclear Research, Esplanade des Particules 1, 1211 Geneva 23, Switzerland
}

(Received 4 November 2021; accepted 22 December 2021; published 18 January 2022)

\begin{abstract}
The electron gun of the REXEBIS charge breeder at the REX/HIE-ISOLDE facility at CERN has been upgraded from a standard magneto-immersed type to a gun using a nonadiabatic magnetic element. The results from the cathode emission and electron beam propagation tests are presented, as well as the charge breeding efficiency for the new design. Complete mass-scans of the extracted beam have been performed from which the level of cathode-originating contaminations could be established, as well as partial pressures of the most abundant residual gases in the ion trapping region. Furthermore, optimal breeding times for a broad range of elements and charge states, either introduced as a gas or externally injected as singly charged ions into the trapping region, are given for different electron currents. From these values, effective electron current densities have been derived. Finally, the axial ion energy distributions of various elements and charge states were also measured, and the derived ion temperatures were correlated with the ion and electron beam overlap factors.
\end{abstract}

DOI: 10.1103/PhysRevAccelBeams.25.013402

\section{INTRODUCTION}

REXEBIS is an electron beam ion source (EBIS) used for charge breeding of radioactive ions [1,2]. After transformation from $1+$ to a higher charge state of choice, the ions are expelled, separated by their mass-to-charge ratio $A / Q$ and subsequently accelerated in a linear accelerator $[3,4]$. The original electron gun was a magneto-immersed gun, with a flat $1.6 \mathrm{~mm}$ diameter cathode positioned in the fringe field of the main solenoid magnet at 2000 Gs. A current of $375 \mathrm{~mA}$ could be extracted although with unstable beam conditions [2]. Moreover, due to lifetime issues the operational value was limited to around $200 \mathrm{~mA}$, yielding a calculated electron current density in the ion trapping region of approximately $100 \mathrm{~A} / \mathrm{cm}^{2}$ by means of adiabatic magnetic compression. In this configuration, REXEBIS has charge bred radioactive beams on around 200 occasions with elements ranging from ${ }^{6} \mathrm{He}$ to ${ }^{228} \mathrm{Ra}$

\footnotetext{
*Heidelberg Graduate School for Physics, Heidelberg University, Im Neuenheimer Feld 226, 69120 Heidelberg, Germany.

'University of Rome 'La Sapienza' and INFN Sez. Roma1, 00185 Roma, Italy.

Centro de Investigaciones Energéticas, Medioambientales y Tecnológicas, Avenida Complutense 40, 28040 Madrid, Spain.

${ }^{\S}$ fredrik.wenander@cern.ch

Published by the American Physical Society under the terms of the Creative Commons Attribution 4.0 International license. Further distribution of this work must maintain attribution to the author(s) and the published article's title, journal citation, and DOI.
}

[5-8]. Yet, there were a number of factors that ultimately warranted an upgrade of the existing electron gun.

Reliability issues of the $\mathrm{LaB}_{6}$ cathodes was one of the reasons for moving to a different type of cathode. After some time of operation, the $\mathrm{LaB}_{6}$ crystals developed cracks near the emission head, leading to fluctuating emission current, and in the worst case detachment of the emission head. Evidence of deterioration can already appear just a couple of months after installation, thereby jeopardizing the experimental run.

More importantly, an increased electron current density in the ion trapping region is desired. As the breeding time is to the first order inversely proportional to the current density, this would lead to shortened hold-up times in REXTRAP (introduced in Sec. IV B) and REXEBIS, and as a result reduced decay losses for short-lived isotopes. In addition, the post-accelerator could then be operated with a higher repetition rate distributing the charge bred particles over more pulses. This is beneficial from an experimental point-of-view as high instantaneous particle rates, potentially leading to pile-up and dead-time in the detectors [9], can be reduced. With the original electron gun, the breeding times for heavy, neutron-rich elements, could be as high as $500 \mathrm{~ms}$. Even for a moderate primary beam intensity and with slow extraction from the EBIS [10] over a pulse length of $1 \mathrm{~ms}$, this yields high instantaneous particle rates $\left(>1 \times 10^{7}\right)$. Finally, an increased repetition rate would also allow for higher particle throughput in the case of high intensity beams.

Apart from reliability and breeding time concerns, the charge breeding efficiency is of utmost importance due to 
the low intensity of the rare isotope beams and should not be lower than in the existing design. Likewise, the residual gas pressure inside the trapping region of the EBIS must not be worsened (e.g., by a higher heat load in the electron collector) as contaminations in the extracted beam can easily submerge the rare radioactive ions. The same requirements hold for atoms evaporated from the hot cathode surface.

To reach higher electron current density with the original gun, the magnetic field in the ion trap would have to be increased proportionally to the desired current density. Such a modification would essentially require building a new ion breeder with a more powerful superconducting solenoid. The implementation of a Brillouin-flow electron gun was considered, for instance the use of the MEDeGUN $[11,12]$. However, since it is not fully tested, particularly in terms of singly charged ion injection efficiency and durability, this approach was rejected. Consequently, we have opted for an improved magneto-immersed electron gun. Combining the immersed gun concept with the technique of a nonadiabatic magnetic field transition, it is feasible to use a high extraction density cathode in a reduced magnetic immersion field. This allowed us to increase the achievable current density while limiting mechanical modifications of REXEBIS to the gun side.

In this paper we present an in-depth report on the design of the new electron gun and the results obtained during the commissioning phase. The following section briefly introduces the concept of nonadiabatic electron beam formation and contains simulation studies of the new gun design, with a particular look at the expected operability under nonoptimal conditions. A description of the mechanical design and assembly of the electron gun is given in Sec. III. The description of the experimental setup is provided in Sec. IV.

Following the installation of the nonadiabatic electron gun, we have run extensive studies to characterize the performance of the upgraded setup which are presented in Sec. V. We present data on the gun perveance and electron beam transmission. This is complemented by charge breeding experiments with various elements. We have validated the throughput efficiency (Sec. VC) of REXEBIS and checked for beam contaminations stemming from both residual gas and the new cathode (Sec. V F). Since measuring the electron beam radius directly would be too invasive and would put the operability of REXEBIS at risk, the effective current density of the new electron beam system has been determined by fitting theoretical charge state evolution models to experimentally recorded charge state spectra, as detailed in Sec. VD. Driven by the observation of a decreasing trend in the effective current density for longer charge breeding times, axial energy spectra have been recorded to characterize the evolution of the ion temperature inside the EBIS. The results of the axial energy studies and a qualitative comparison to the current density results are laid out in Sec. V E.

\section{ELECTRON BEAM SIMULATION}

\section{A. Concept and simulation model}

In Ref. [13], it was concluded that the energy of the cyclotron motion for charged particles in coherent beams can be decreased using a nonadiabatic (NA) magnetic field. By situating a local magnetic field depression of the main guiding field in the region of the descending phase of the beam oscillation, the cyclotron motion can be reduced. The required local magnetic field depression in the REXEBIS case is generated with a soft iron ring, the so-called NA element. By applying this method, we can create ripple-free laminar beams, even for magneto-immersed guns positioned in a magnetic field of only a few hundred Gs and with a cathode emission current density exceeding $20 \mathrm{~A} / \mathrm{cm}^{2}$. Consequently, a high magnetic compression and beam current density in the full field region can be attained, without electrons reflecting when entering the field of the main solenoid.

The REXEBIS electron gun is of Pierce-type with electrostatic focusing field inside the cathode-anode gap. The flat cathode diameter is $2.0 \mathrm{~mm}$, and the simulated perveance is $0.73 \mu \mathrm{A} / \mathrm{V}^{3 / 2}$. Its basic geometry was described in Ref. [13] and can be seen in Fig. 1. As the charge breeding efficiency is of paramount importance, the 1+ ion acceptance of the new electron beam must be maintained. Thus, with a smaller electron beam radius in the trapping region, the electron current must be increased with respect to the old gun design. Using the analytic formula for transverse acceptance given in Ref. [14], an acceptance of $10 \mu \mathrm{m}$ is calculated for ${ }^{39} \mathrm{~K}^{+}$at $30 \mathrm{keV}$, with electron beam characteristics of $200 \mathrm{~mA}$, $5400 \mathrm{eV}$ and $250 \mu \mathrm{m}$ beam radius. For the NA gun with a simulated electron beam radius of $187 \mu \mathrm{m}$ in full field, a current of $500 \mathrm{~mA}$ at $6400 \mathrm{eV}$ is required to reproduce the same acceptance.

The electron beam simulations reported in this paper have been carried out with the solenoid geometry of REXEBIS. The electron gun is positioned in the stray field of the iron-shielded superconducting solenoid, which has a magnetic field of $B=2.0 \mathrm{~T}$ in its center. For the simulations we used the 2.5D TRAK charged particle toolkit from Field Precision LLC [15] in most cases. For

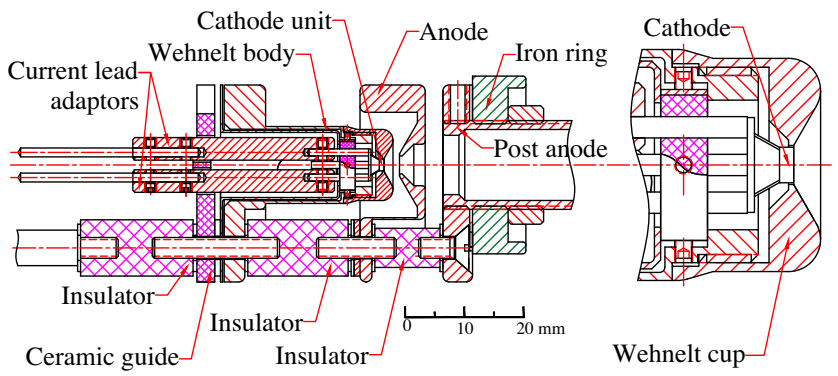

FIG. 1. Left: design of REXEBIS electron gun with an $\mathrm{IrCe}$ cathode and iron ring as NA element. Right: enlarged view showing the cathode unit and Wehnelt. 
the purpose of cross-verification, some additional full 3D simulations were performed with CST Particle Studio [16], and they exhibit good agreement. Due to practical limitations concerning the runtime and memory use of the simulations, the beam was not tracked all the way into the full magnetic field region but up to an axial field of approximately $1.7 \mathrm{~T}$. Since we observe a good adherence to the predictions of the Herrmann theory [17] in the simulated interval, we expect that the beam properties in full magnetic field can be extrapolated safely with the established formalism.

Based on our current density and acceptance requirements we have decided to optimize the geometry of the electron gun for an operation with $500 \mathrm{~mA}$ beam current with a residual magnetic flux density of approximately 700 Gs on the cathode surface. Below, we present simulations for various beam currents and show the effect of an axial misalignment of the gun, as well as a mitigation strategy. Furthermore, we have considered the possibility of further reducing the magnetic flux density on the cathode in order to optimize the beam compression.

\section{B. Launching of electron beams with different currents}

The following series of simulations were performed with the purpose of determining the dynamic electron current range of the NA gun, located at a position corresponding to a 700 Gs magnetic stray field of the superconducting solenoid, and with an NA element optimized for an electron current of $500 \mathrm{~mA}$. The ability to run the electron gun at different beam currents is crucial, especially when cathode limitations or operational considerations mandate a reduction of the beam current.

With a Child-Langmuir model applied to the electron emission, we used the cathode-to-anode voltage to control the beam current in our simulations. The envelopes of the electron beam for different electron beam currents are presented in Fig. 2. One can see that deviations of the electron current from the optimum value result in an increased amplitude of radial beam oscillations. Nevertheless, for an electron current in the range of $300 \mathrm{~mA}$ to $800 \mathrm{~mA}$, the radial ripples of the beam do not exceed $15 \%$ if the electron energy is optimized. The reason for increased beam oscillations when the electron current deviates from the optimized value of $500 \mathrm{~mA}$ is a change in phase of the cyclotron oscillation at the location of the NA element: for reduced currents, when the anode voltage is lower, the oscillation phase occurs earlier and for the higher electron current, when the anode voltage is higher, the oscillation phase appears later. We have previously shown that the oscillatory behavior of the beam in this situation shows an excellent agreement with the immersed laminar beam theory [13], increasing the confidence in our simulation results.

To reduce the sensitivity to the electron energy variation, the anode is made as short as possible, and the phase of the

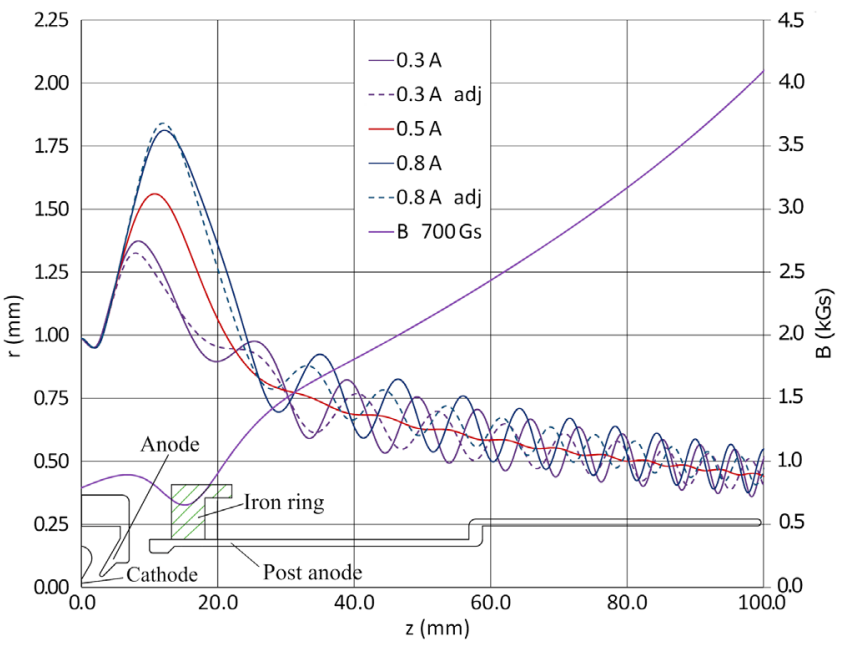

FIG. 2. Electron beam envelopes for beam currents of $300 \mathrm{~mA}$, $500 \mathrm{~mA}$, and $800 \mathrm{~mA}$. The magnetic flux density at the cathode is $\approx 700 \mathrm{Gs}$ and the purple curve shows its value along the $z$-axis. Solid lines represent the beam envelope for a fixed potential difference between the cathode and the post anode of $7.8 \mathrm{kV}$, while the dotted lines are for an adjusted potential difference between the cathode and the post anode $(10.8 \mathrm{kV}$ for $0.3 \mathrm{~A}$ and $6.8 \mathrm{kV}$ for $0.8 \mathrm{~A}$ ). NB: The vertical dimensions of the machine geometry are not to scale.

oscillation can be adjusted by varying the potential of the post anode tube. The envelopes of the electron beam for electron currents $300 \mathrm{~mA}$ and $800 \mathrm{~mA}$ with adjusted electron energy in the post anode tube are shown in Fig. 2 with dotted lines. Our simulations demonstrate that by optimizing the electron energy it is possible to reduce the amplitude of radial oscillations. This reduction depends on the electron current: for $0.3 \mathrm{~A}$ it is $6.4 \%$ and for $0.8 \mathrm{~A}$ it is $47 \%$. To reach this reduction, the potential on the post anode drift tube has to be increased by $3 \mathrm{kV}$ for an electron current of $0.3 \mathrm{~A}$ and reduced by $1 \mathrm{kV}$ for a current of $0.8 \mathrm{~A}$.

From our simulations we extrapolate an electron beam radius of $(187 \pm 1) \mu \mathrm{m}$ for a beam energy of $7.8 \mathrm{keV}$ in the full $2.0 \mathrm{~T}$ field and currents between $200 \mathrm{~mA}$ and $800 \mathrm{~mA}$. For the nominal beam current of $500 \mathrm{~mA}$ this translates to a current density of $\approx 450 \mathrm{~A} / \mathrm{cm}^{2}$. Limited by cathode emission, our experiments were mostly performed at beam currents of $200 \mathrm{~mA}$ and $300 \mathrm{~mA}$, corresponding to current densities of $180 \mathrm{~A} / \mathrm{cm}^{2}$ and $270 \mathrm{~A} / \mathrm{cm}^{2}$, respectively.

\section{Electron beam oscillation dependence on axial shift of the electron gun and NA element}

Another simulation series was performed to study the sensitivity of the electron beam quality on the axial position of the electron gun assembly, which includes the gun itself and the iron ring, and the possible mitigation of oscillations introduced with such a shift. The simulations were performed for a fixed electron current of $500 \mathrm{~mA}$. As expected, an axial shift of the electron gun from its optimal position in 


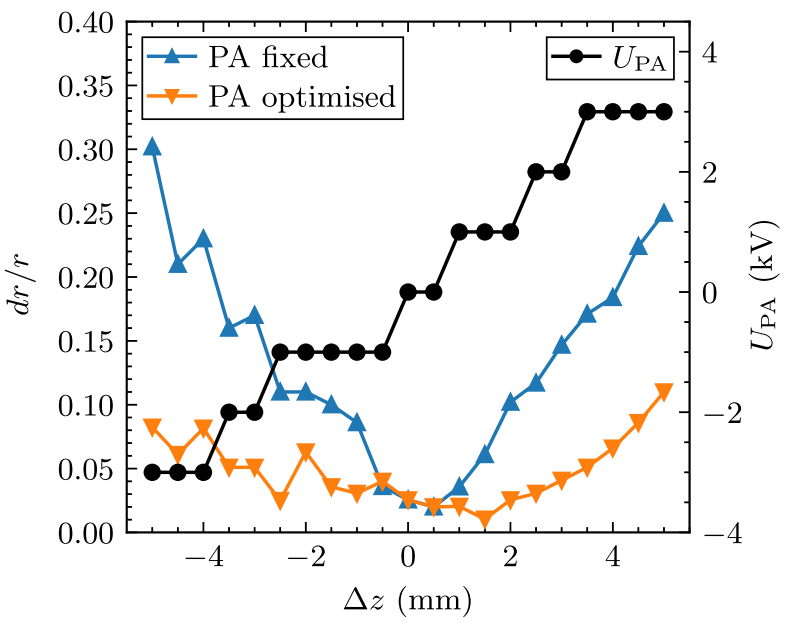

FIG. 3. Dependence of the relative electron beam oscillation on the shift of the electron gun and iron ring from the optimum position for: (blue) a fixed potential on the post anode (PA) $\left(U_{\text {post anode }}=0 \mathrm{~V}\right)$; (orange) optimized post anode potential. The post anode potential for the optimized case is presented with the black curve.

the magnetic fringe field of the superconducting solenoid results in increased oscillations of the beam as the oscillation-compensating NA element ends up at the wrong phase of cyclotron oscillations. Using the same method as described above, the average velocity of the electrons from the cathode to the iron ring can be controlled by applying different voltages to the post anode, which holds the iron ring. Figure 3 demonstrates the effect of such a mitigation. Here, the coarse steps of the optimized post

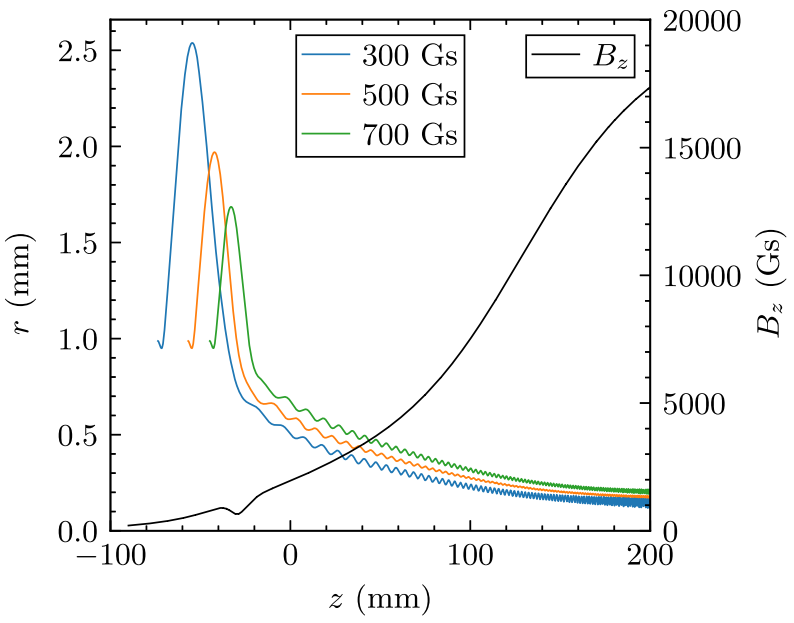

FIG. 4. Envelopes of a $700 \mathrm{~mA}$ electron beam generated with the NA electron gun positioned at different magnetic field strengths. The shown magnetic field distribution (black), with the dip at the axial position of the NA element, has been optimized for a cathode positioned for a cathode flux density of 700 Gs. anode voltage are due to the run time of the simulations; in practice this voltage can be tuned continuously.

\section{Variation of the cathode field}

In anticipation of future upgrades, we have also explored the achievable limits of the nonadiabatic electron beam injection technique during the simulation campaign.

By pushing the electron gun further out of the fringe field, the magnetic flux density at the cathode has been reduced to as little as 360 Gs for an electron current of $700 \mathrm{~mA}$. We have found that by adapting the magnetic field gradient through a tuning of the position and geometry of the nonadiabatic element, the ripple amplitude of the beam can still be maintained at a low level. A comparison of three beam envelopes launched in different magnetic fields is shown in Fig. 4. An unavoidable side effect of the reduced magnetic field is a weaker confinement of the electron beam compared to the constant space charge effects. This causes an increase of the amplitude of the first oscillations prior to passing the nonadiabatic element, which may eventually require an adaptation of the gun aperture. Nevertheless, the simulation results are encouraging as they suggest that this method offers further room for improving the beam compression in the future. The simulated current densities for a range of magnetic field values are shown in Fig. 5 together with extrapolated values for the full magnetic field, and a theoretical prediction obtained with the Herrmann formula [17].

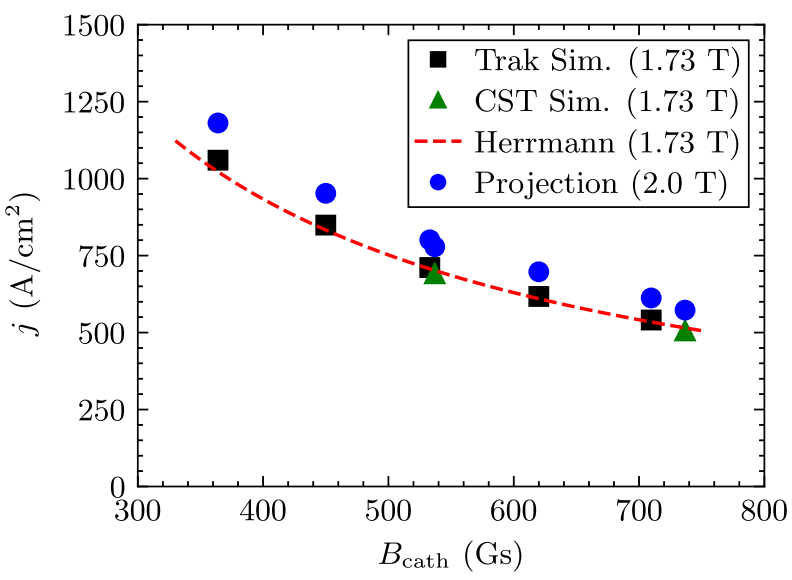

FIG. 5. Dependence of simulated electron beam current density versus magnetic field on the cathode surface of the NA gun. The electron current is $0.7 \mathrm{~A}$ and the magnetic field at the end of the simulation model is $1.73 \mathrm{~T}$. Tracking simulation results are marked with black squares (TRAK) and triangles (CST). The corresponding current densities predicted by the Herrmann theory are indicated with a dashed red line. The blue dots mark the extrapolated current density inside the full magnetic field of $2 \mathrm{~T}$. 


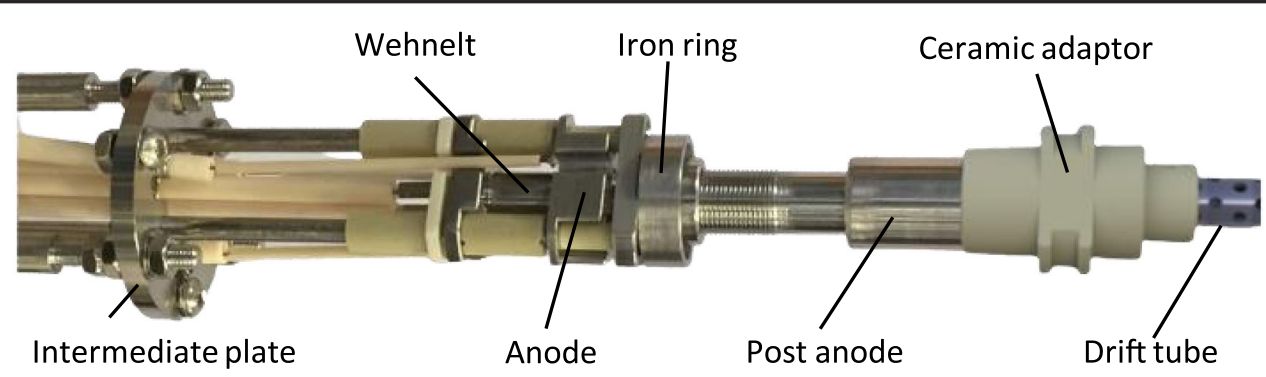

FIG. 6. Photograph of the NA electron gun assembly with the threefold symmetric support and intermediate plate to the left, and to the right the isolating ceramic adapter piece aligning the gun to the first drift tube.

\section{MECHANICAL DESIGN}

\section{A. General layout}

The new electron gun design was adapted to the existing tight space constraints of REXEBIS. A minimum impact on the internal structure, affecting primarily the electron gun region, was aimed for. Apart from the electron gun itself, the main changes entailed an axial displacement of the cathode emission surface to a lower magnetic field (700 Gs instead of $2000 \mathrm{Gs}$, as in the original electron gun) and the introduction of a post anode electrode, thus the support structure and the connection interface to the drift tubes structure were remade. A cross section showing the actual electron gun with the main elements indicated is found in Fig. 1.

The Wehnelt electrode is isolated from the cathode, which gives some flexibility for focal tuning of the electron beam. The potentials of the anode and post anode are individually controlled. The electrodes are physically assembled with steatite standoffs, providing sufficient creeping distance along the surfaces for voltages differences up to $19 \mathrm{kV}$ between cathode and anode, and $11 \mathrm{kV}$ between anode and post anode. Special attention has been paid to increase sparking distances, and as a result the physical geometry of the Wehnelt body is quite intricate. The emitting surface of the cathode is flat, like in the previous version, with a diameter of $2 \mathrm{~mm}$. The electron gun does not feature water cooling as the heating power for the cathode was estimated to be below $20 \mathrm{~W}$ and the electron beam losses on the anode were expected to be negligible. In the end, we operate with a cathode heating power close to $30 \mathrm{~W}$.

\section{B. Mechanical structure}

The Wehnelt, anode and post anode have almost a threefold rotational symmetry, as seen in Fig. 6 that presents a photo of the gun assembly including the support structure and interface to the drift tube. Thus, the gun is supported by three solid stainless steel rods that, via an intermediate plate, attach the gun to the CF63 end flange at the electron gun vacuum cross. By means of the intermediate plate, the axial position of the gun can easily be adjusted as the post anode is free to move axially within the ceramic adaptor. Likewise, the axial position of the iron ring with respect to the cathode can be fine-adjusted as it is threaded onto the post anode electrode. The radial alignment of the electron gun assembly with respect to drift tube structure is assured by a narrow tolerance $( \pm 50 \mu \mathrm{m})$ between the ceramic adaptor and the first drift tube. Once engaged, the combined electron gun and drift tube structure can be adjusted radially with respect to the magnetic field axis as the vacuum chamber of REXEBIS is detached from the magnet bore.

As the radial and axial mechanical tolerances of the $\mathrm{IrCe}$ cathode surface with respect to the mounting base was not better than a couple of hundred $\mu \mathrm{m}$ (established by $\mathrm{x}$-ray tomography of two IrCe units), the gun design has to allow for radial adjustment of the cathode (see Fig. 7). The concentricity between cathode and Wehnelt hole is better than $30 \mu \mathrm{m}$. Furthermore, the cathode should be recessed inside the Wehnelt by $50 \mu \mathrm{m}$ to $100 \mu \mathrm{m}$ to mitigate undesirable side emission from the cathode cylinder. In principle, the cathode-to-Wehnelt distance can be varied by means of the Wehnelt cup threaded on to the Wehnelt body, but the adjustment was finally done by placing a $100 \mu \mathrm{m}$ shim of tantalum foil between the cathode and the interior mating surface of the Wehnelt body.

\section{Material selection and material treatment}

The electrodes are produced from non-magnetic stainless steel (316LN). The soft iron ring, made of steel 1010, was annealed to improve the homogeneity of the magnetic permeability. In case of a future upgrade to a dispenser cathode (see below), an adapted Wehnelt piece and anode

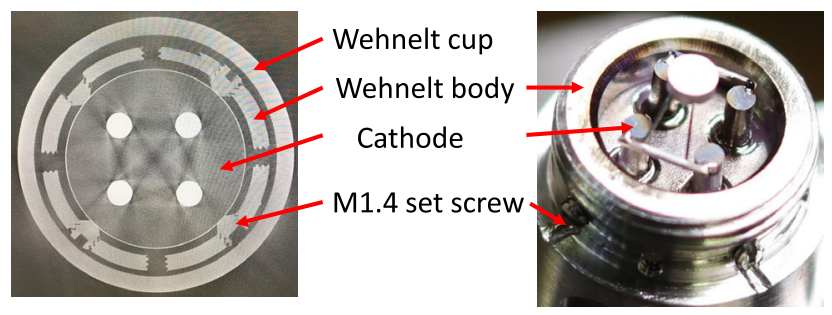

FIG. 7. IrCe cathode mounted inside the Wehnelt body. Left: x-ray tomography picture of the assembly showing the cathode base with four connection rods providing the current to the cathode heating, the set screws for radial positioning, as well as Wehnelt body and cup. Right: photograph of the same assembly, but with the Wehnelt cup removed. 
made of molybdenum will be utilized to minimize the risk of cathode surface poisoning. Due to logistic complications the metal parts were not vacuum fired before insertion, but the electron gun was baked out at $300^{\circ} \mathrm{C}$ for $37 \mathrm{~h}$ after installation. The isolating standoffs are of steatite type, while the ceramic adapter is made of $\mathrm{Al}_{2} \mathrm{O}_{3}$. The latter was heat fired in an air atmosphere. As the $\mathrm{IrCe}$ cathode contained an insulating material of undisclosed composition, a residual gas analysis (RGA) was performed on a cold cathode, showing no outgassing above background level for a baked out RGA system.

\section{Choice of cathode type}

To attain a beam current of $500 \mathrm{~mA}$ from a $2 \mathrm{~mm}$ diameter cathode, an electron emission of about $16 \mathrm{~A} / \mathrm{cm}^{2}$ is required. This is a high value for an emitter operating in continuous mode for up to nine months without exchange. A low evaporation rate is therefore imposed, but also of importance from an ion beam contamination point-of-view. Otherwise, evaporated cathode material may effuse into the ionization region and overlap charge bred radioactive beam at certain $A / Q$-values. In the original REXEBIS electron gun, a single-crystal $\mathrm{LaB}_{6}$ cathode had been employed. The $\mathrm{LaB}_{6}$ cathode is advantageous as La has only a single stable isotope at $A=139$ and ${ }^{10,11} \mathrm{~B}$ can in many cases be suppressed from the extracted beam by stripping foils in the accelerating linac. The clamping and heating arrangement of the $\mathrm{LaB}_{6}$ cathode, however, limits the long-term operation to $\approx 10 \mathrm{~A} / \mathrm{cm}^{2}$, corresponding to an emitted current of $200 \mathrm{~mA}$ for the $1.6 \mathrm{~mm}$ diameter cathode.

A high electron emission in excess of $20 \mathrm{~A} / \mathrm{cm}^{2}$ at $1850 \mathrm{~K}$ has been reported for IrCe alloy cathodes [18], although earlier tests at the TwinEBIS test bench at CERN only reached $\approx 15 \mathrm{~A} / \mathrm{cm}^{2}$ at this temperature [19]. Nonetheless, for the initial tests of the NA electron gun an $\mathrm{IrCe}$ cathode was employed. According to the manufacturer, a lifetime exceeding $5000 \mathrm{~h}$ can be attained if operated with an emission of $500 \mathrm{~mA}$ to $600 \mathrm{~mA}$ [20], and literature states a low evaporation rate [21].

Tests with a nanosized-scandium-doped impregnated cathode [22] are also foreseen and the necessary modifications of the gun electrodes are under preparation. This cathode type has demonstrated a current density of $30 \mathrm{~A} / \mathrm{cm}^{2}$ at $950^{\circ} \mathrm{C}$, for more than $2000 \mathrm{~h}$ of continuous operation.

A concern for the two latter cathodes is the large number of stable isotopes that make up the cathode material, for instance ${ }^{191,193} \mathrm{Ir},{ }^{140,142} \mathrm{Ce}$ and ${ }^{45} \mathrm{Sc},{ }^{135,136,137,138} \mathrm{Ba}$, $182,183,184,186 \mathrm{~W}$. Special attention to this concern was paid during the commissioning phase (see Sec. VF).

\section{EXPERIMENTAL SETUP}

\section{A. REXEBIS layout and operational conditions}

The full testing and commissioning of the NA electron gun was carried out at REXEBIS. A detailed description of the original EBIS is found elsewhere $[1,23]$. Figure 8 shows a schematic layout of the key elements of the ion source, the typical electrical potentials when operating with the new gun, and relative position within the magnetic field. The $80 \mathrm{~cm}$ long trapping region and the barriers are located in full magnetic field. The outer barrier is pulsed between $600 \mathrm{~V}$ (injection), $1300 \mathrm{~V}$ (breeding) and $0 \mathrm{~V}$ (extraction). The 7 perforated drift tubes have an inner radius of $5 \mathrm{~mm}$. With a combination of turbo pumps located at the gun and collector vacuum crosses and NEG strips surrounding drift tube area, average pressures of approximately $1 \times$ $10^{-10}$ mbar to $1 \times 10^{-9}$ mbar were recorded at the gun and collector crosses, respectively, during the commissioning campaign.

\section{B. Ion injection test setup}

For the ion injection tests, the complete REX low-energy stage was exploited $[4,24]$. A layout of the setup is shown in Fig. 9. REXEBIS is preceded by REXTRAP [25], a large, Ne gas-filled Penning trap used for accumulation, cooling and bunching of the $1+$ beam to be injected into the breeder. The expelled pulses have a FWHM length of $1 \mu \mathrm{s}$ (Li) to $10 \mu \mathrm{s}(\mathrm{Tl})$, shorter than the ion round-trip time inside the REXEBIS trapping region, and thus allows for pulsed injection into the synchronized breeder. An electrostatic beam transfer line connects REXTRAP with REXEBIS. Two focusing Einzel lenses and two pairs of transverse steerers are located in front of REXEBIS (see Fig. 9 in Ref. [26]). These elements are pulsed, permitting different settings to be applied during injection and extraction. The beam extracted from REXEBIS passes a Nier-type spectrometer for $A / Q$-selection [27].

The $1+$ ions were produced either in a local surface ion source [28] positioned upstream of REXTRAP (for $\mathrm{K}^{+}$and $\mathrm{Cs}^{+}$) or at the ISOLDE General Purpose Separator [29] (for $\mathrm{Li}^{+}, \mathrm{Na}^{+}, \mathrm{Sm}^{+}$and $\mathrm{Tl}^{+}$). Neutral Xe gas was injected via a precision leak valve located at the electron gun vacuum cross, while for the $\mathrm{Ne}$ studies gas effusing from REXTRAP was used. The ion intensities during the tests were low, at most $2.5 \times 10^{7}$ ions/bunch injected into REXTRAP, and the space charge compensation of the REXEBIS electron beam from the injected ions at the instance of extraction was less than $2.5 \%$. The exception was for some Xe measurements, where a neutralization degree of $7.5 \%$ was attained for $T_{\text {breed }}=90 \mathrm{~ms}$. Additional studies of space charge compensation from residual gases and cooling gas from REXTRAP are presented in Sec. V F.

The cooling and transmission conditions inside REXTRAP were specifically optimized for each singly charged target ion species. The period time varied between $20 \mathrm{~ms}$ (lower limit imposed by the cooling time inside the trap) and $600 \mathrm{~ms}$ (required breeding time to reach the highest charge states for ${ }^{205} \mathrm{Tl}$ ). Furthermore, as the transfer time between the Penning trap and EBIS varies with the ion mass, the exact closing time of the EBIS outer trap barrier 


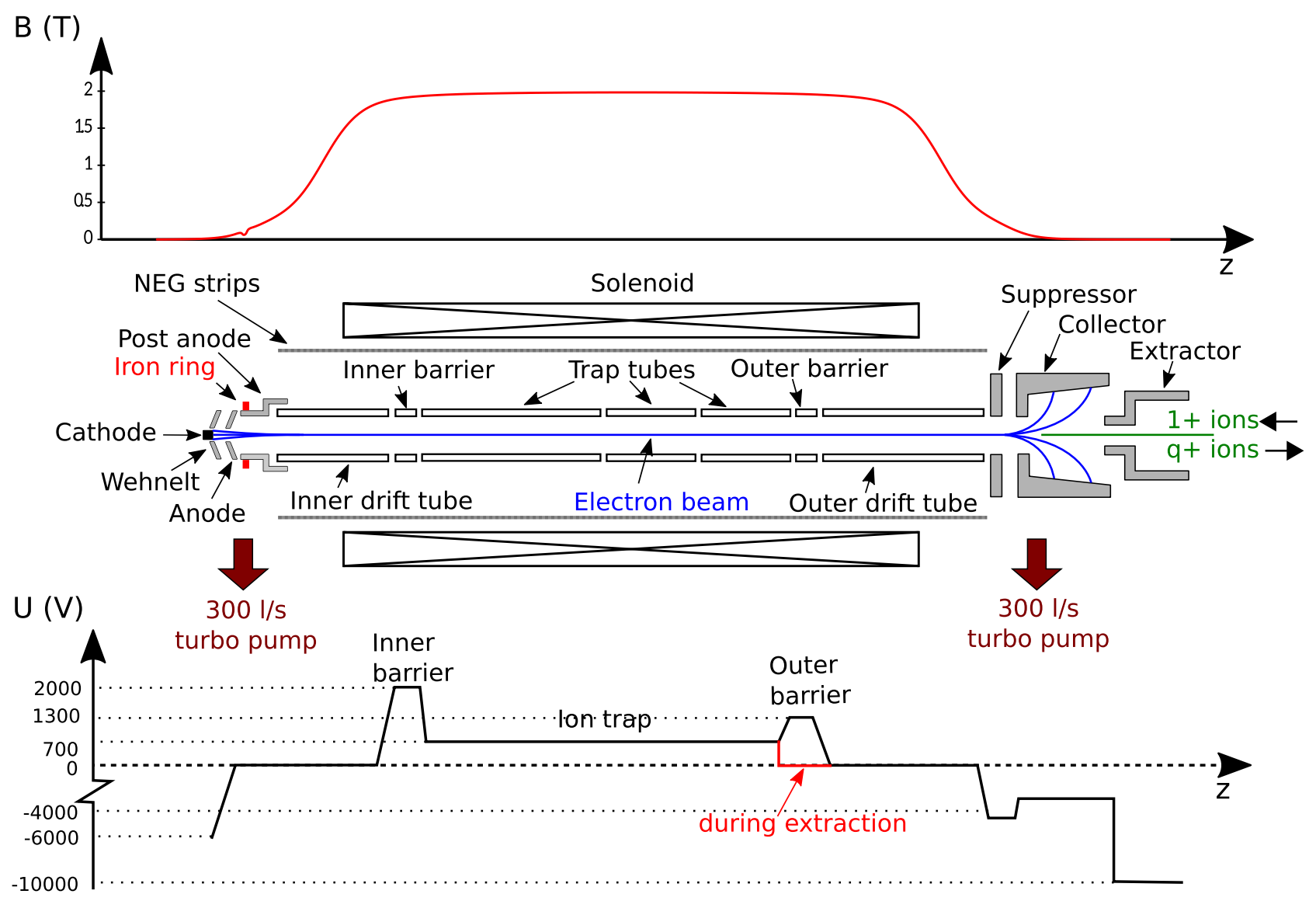

FIG. 8. Schematic view of REXEBIS with the NA electron gun installed. The axial magnetic field strength and potentials on the electrodes are indicated. All voltages are given with respect to the EBIS platform potential. The dimensions are not to scale.

with respect to the expulsion moment from the Penning trap was tuned for the best ion injection. As the breeding efficiency was maximized, the total ion energy when entering the EBIS trapping region was found to vary between $200 \mathrm{eV}$ to $300 \mathrm{eV}$ for different ion types and injection conditions. The $1+$ ion current can be observed at Faraday cups FC1 and FC2 before and after REXTRAP, respectively; thereafter the non-separated beam from the EBIS is measured with FC3, and finally the separated beam at FC4 after the separator magnet (see Fig. 9 for FC positions). The efficiencies discussed in Sec. VC, for an element being charge bred to $Q$, are defined as

$$
\begin{gathered}
\eta_{\text {single }}(Q)=\frac{F C 4(Q)}{F C 2} \\
\eta_{\text {global }}=\frac{\sum_{Q=1}^{Z} F C 4(Q) / Q}{F C 2},
\end{gathered}
$$

where $F C x$ denotes time-averaged current readings at the different Faraday cups. The degree of space charge neutralization of the electron beam is defined as

$$
\eta_{\text {neutralization }}=\frac{F C 3 \cdot T_{\text {period }}}{L_{\text {trap }} I_{e} / v_{e}}
$$

where $L_{\text {trap }}, I_{e}$ and $v_{e}$ denote trap length, current and electron beam velocity inside the EBIS. $T_{\text {period }}$ denotes the

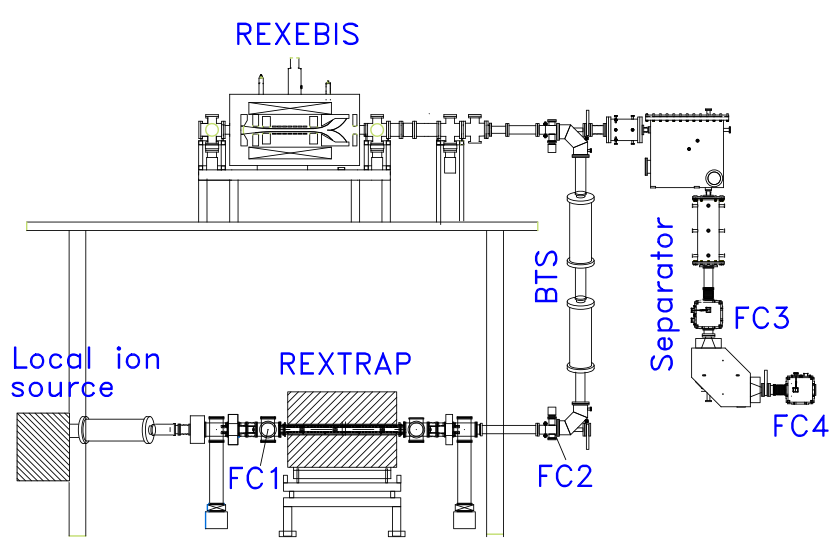

FIG. 9. Layout of the REX low-energy stage. The transfer lines are fully electrostatic and the $A / Q$-separation takes place in the separator magnet between FC3 and FC4. With a kicker element at the top of the BTS section, the extracted ion beam can be sent to the separator section. 
period time of the entire cycle of the REX setup, and should not be confused with the breeding time which is always smaller than the period time. The electron velocity has been computed by taking into account the beam induced space charge and iteratively finding a self-consistent solution for the associated equations

$$
\begin{gathered}
v_{e}=\sqrt{\frac{2 e\left(U_{\text {trap tube }}-U_{\text {cathode }}+\Phi(0)\right)}{m_{e}}} \\
\Phi(0)=\frac{I_{e}}{4 \pi \epsilon_{0} v_{e}}\left(2 \ln \left(\frac{r_{\text {tube }}}{r_{\text {beam }}}\right)+1\right)
\end{gathered}
$$

where $m_{e}$ denotes the electron mass, and $r_{\text {beam }}$ and $r_{\text {tube }}$ the electron beam and drift tube radii in the trapping region. Used numerical values are $U_{\text {trap tube }}=700 \mathrm{~V}, 200 \mathrm{~mA}<I_{e}<$ $300 \mathrm{~mA},-6300 \mathrm{~V}<U_{\text {cathode }}<-5700 \mathrm{~V}$ (depending on electron beam current), $r_{\text {beam }}=187 \mu \mathrm{m}$ and $r_{\text {tube }}=5 \mathrm{~mm}$.

\section{EXPERIMENTAL RESULTS}

\section{A. Gun perveance}

The gun perveance measurement was carried out by recording the extracted current as a function of cathode-toanode voltages up to $1400 \mathrm{~V}$. A perveance of $0.866 \pm$ $0.007 \mu \mathrm{A} / \mathrm{V}^{3 / 2}$ was measured, see Fig. 10, to be compared to a simulated design perveance of $0.73 \mu \mathrm{A} / \mathrm{V}^{3 / 2}$. Here, the perveance error is the uncertainty of the fit parameter. Side emission from the cathode and a possibly shorter cathodeto-anode distance than the design value may explain the discrepancy. The range of the perveance measurement was limited to low currents, due to issues with the cathode and loss currents detailed in the following section.

\section{B. Electron beam current and losses}

Our simulations of the electron beam dynamic range with the NA electron gun in the Child-Langmuir regime indicate, that with an increased deviation from the optimum

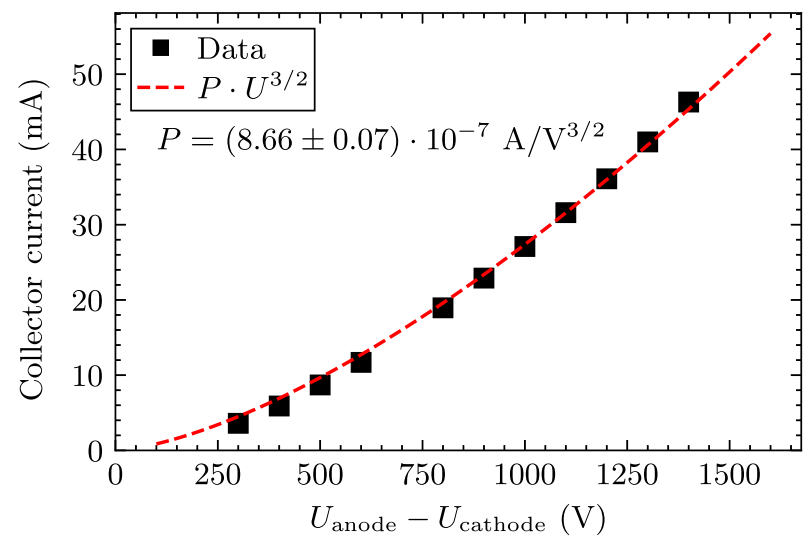

FIG. 10. Measured emission current as a function of cathodeto-anode voltage, with fitted experimental perveance value. electron beam current of $500 \mathrm{~mA}$, the amplitude of radial oscillations increases (Fig. 2). Nevertheless, in our experiments, we managed to propagate an electron beam with current ranging from a minimum of few $\mathrm{mA}$ to $420 \mathrm{~mA}$. The gun was constantly operated in the thermionically limited regime due to inadequate emission, and the electron current was adjusted by the cathode heating current while the anode voltage was kept fixed. Apart from tuning the electrical potentials, the electron beam losses were minimized by adjusting the transverse position of the electron gun, drift tube and collector ensemble at the gun and the collector crosses with respect to the magnetic field axis.

Figure 11 shows the beam losses, reaching the anode electrode and the outer drift tube in front of the suppressor, as a function of the emitted current. Up to a current of $300 \mathrm{~mA}$ reaching the collector, the voltage settings were kept unchanged $\left(U_{\text {cathode }}=-6 \mathrm{kV}, U_{\text {anode }}=0 \mathrm{~V}\right.$, $U_{\text {post anode }}=0 \mathrm{~V}, U_{\text {outer tube }}=0 \mathrm{~V}, U_{\text {suppressor }}=1.7 \mathrm{kV}$, and $U_{\text {collector }}=2.5 \mathrm{kV}$ ). Above $300 \mathrm{~mA}$, the settings were tuned in order to minimize the losses, in particular $U_{\text {gun }}$ was increased to $-7 \mathrm{kV}$ while $U_{\text {suppressor }}$ was lowered to $1.3 \mathrm{kV}$. The post anode has no positive influence when operating at higher currents. The current loss to the anode is very low, whereas the loss to the outermost drift tube accounts for approximately $0.03 \%$ of the emitted current.

Instead of permanently operating the outer drift tube at $0 \mathrm{~V}$, a pulsed voltage supply was connected to the electrode. The bias voltage was applied for $48 \mathrm{~ms}$ and thereafter reduced to $0 \mathrm{~V}$ for $2 \mathrm{~ms}$ while the ions were expelled from the trapping region. The average loss current to the outer drift tube was reduced, although only by approximately $10 \%$ if $+3500 \mathrm{~V}$ was applied. With a negative pulsed voltage applied to the electrode, the losses increased. To launch the electron beam, the electron gun voltage has first to be applied, and thereafter the cathode heated to initiate the emission. The other way round, excessive anode losses

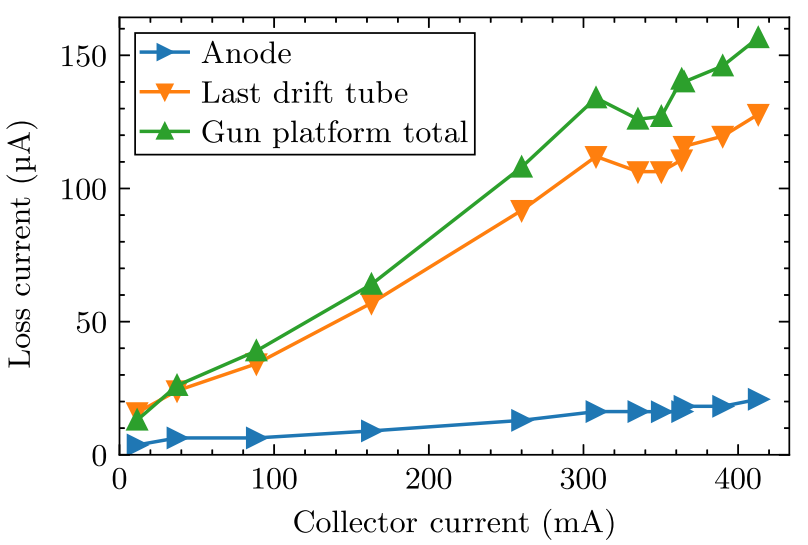

FIG. 11. Measured losses as a function of the collector current. For collector currents above $300 \mathrm{~mA}$, the voltage settings were adjusted to minimize the losses. 
in the intermediate gun voltage region $(>10 \mathrm{~mA}$ between $2000 \mathrm{~V}$ to $4500 \mathrm{~V}$ ) prevents the launch of the beam.

For electron beam currents up to $400 \mathrm{~mA}$, the electron losses are acceptable, even though neither the anode nor the last drift tube are water cooled. Reducing the losses further would therefore improve the vacuum conditions. The low fraction of beam being intercepted by the anode does suggest that the electron gun design is sound, and that the cause of the losses has to be found elsewhere. As the electron beam losses are monotonically decreasing with a reduced suppressor electrode voltage, it is believed that elastically reflected and/or backscattered electrons from the collector region are the culprit. On the other hand, with a negative voltage applied to the outer drift tube, one would expect a further reduction in losses, which was not observed. Tests at a higher electron current are foreseen in the future after upgrading to a dispenser-type cathode, see also Sec. VI.

\section{Ion breeding efficiency \\ 1. Breeding efficiency methodology}

The ion breeding efficiency (defined in Sec. IV B) of REXEBIS while being equipped with the NA electron gun, was measured for a group of ions $\left({ }^{7} \mathrm{Li},{ }^{23} \mathrm{Na},{ }^{39} \mathrm{~K},{ }^{152} \mathrm{Sm}\right.$ and ${ }^{205} \mathrm{Tl}$ ) at various charge states and for different electron currents. Either the breeding time was adjusted for the element and charge state in question to attain maximum current, or the charge state distributions were recorded for fixed breeding times. In the latter case, the recorded EBIS single charge state efficiencies may not have been at their optimal values. For each element and electron beam current, the ion injection conditions, such as beam focusing, steering and injection energy, were adjusted. The main uncertainty factors are a suboptimal optics tuning that may introduce a varying degree of ion losses between the test elements, and high relative current error readings after REXTRAP for sub-pA average currents. We adopt a $1 \sigma$ measurement error comprised of an absolute error of $0.1 \mathrm{pA}$ and an additional relative error of $5 \%$ of the measured ion current. Large error bars in connection with the ${ }^{23} \mathrm{Na}$ data, caused by a systematic error (see Sec. V C 3), have been excluded in general for visual clarity reasons.

\section{Electron beam influence on breeding efficiency}

Throughout the investigation, it was observed that even small changes to the drift tube settings, particularly the post anode and first drift tube voltages, have an influence on the breeding efficiency. Also, small adjustments to the electron beam energy affects the efficiency, even in regions with little cross section dependence on the beam energy. During the electron current transmission measurements, it was noted that fine-tuning of these voltages has an influence on the electron loss current. Hence, it is believed that the electron beam propagation in the collector region is modified, which may affect the $1+$ ion acceptance.

\section{Global efficiency results}

The EBIS global efficiency was recorded for some of the elements and is presented in Fig. 12. Most of the curves lie within a band between $65 \%$ to $85 \%$. The notably lower global efficiency for $\mathrm{Na}$ is believed to be caused by a systematic error in the REXTRAP current readout that may appear if $\mathrm{Ne}^{+}$ions are created and expelled onto the current detector due to unfavorable ion injection conditions into the trap. No statistically significant drop in global efficiency with breeding time is observed, suggesting that ion losses are negligible during the breeding process. The global efficiencies are also found consistent between $200 \mathrm{~mA}$ to $300 \mathrm{~mA}$ electron currents. Finally, no mass-correlation is seen as light Li and heavy elements exhibit similar global efficiency values. The large error bars for $\mathrm{Li}$ and data points with long breeding time are ascribed to very low average beam intensities leaving REXTRAP.

\section{Single charge state efficiency results}

The EBIS single charge state efficiency is presented in Fig. 13 for an electron beam current of $200 \mathrm{~mA}$. Several features are noteworthy, for instance the closed atomicshell effect, enhancing the efficiency in a single charge state for $\mathrm{Li}^{3+}$ (fully stripped), $\mathrm{Na}^{9+}$ (He-like) and $\mathrm{K}^{9+, 17+}(\mathrm{Ne}-$ and He-like). For the Sm case the $3 d$ orbital closure occurs for $34+$, which was not explicitly recorded during the measurement, although an increase in the occupancy of the near-lying $36+$ charge state is visible. The low efficiency for ${ }^{39} \mathrm{~K}^{8+}$ is not in accordance with the relative abundance prediction from charge state simulations but has been confirmed on several measurement occasions. It is related to the imminent shell closure and briefly discussed in Sec. VD 3.

The increased single charge state efficiency for highly charged $\mathrm{Tl}$ ions is caused by the ionization potentials

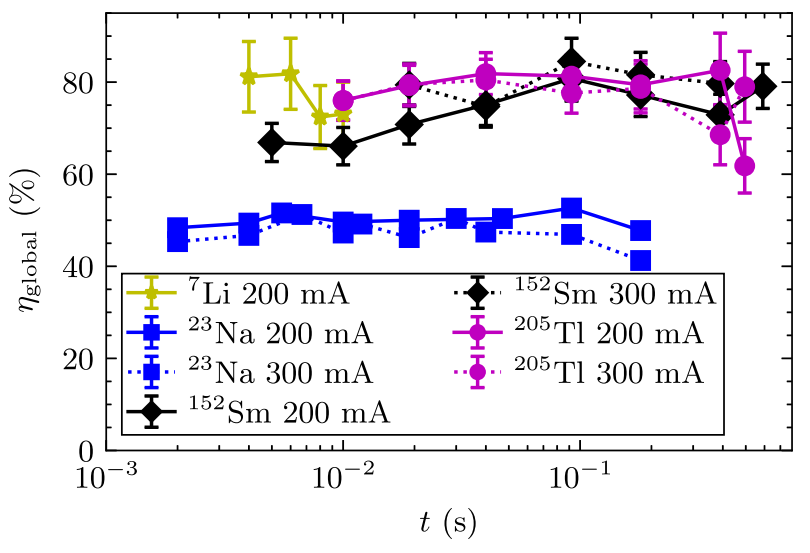

FIG. 12. EBIS global efficiency as a function of breeding time for different elements. Systematic errors are not indicated. 


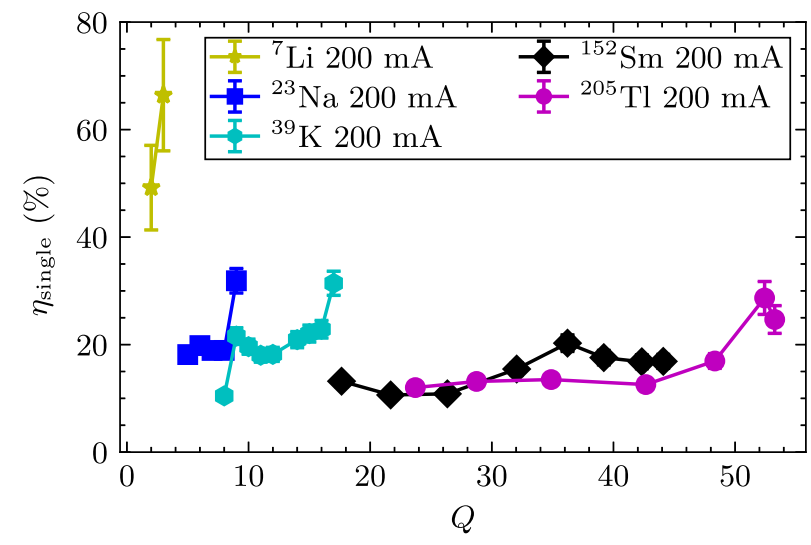

FIG. 13. EBIS single charge state efficiencies as a function of average charge state for different elements and an electron beam current of $200 \mathrm{~mA}$.

approaching the electron beam energy $(6100 \mathrm{eV})$, for instance $I P\left(\mathrm{Tl}^{53+}\right)=4854 \mathrm{eV}[30]$, thereby reducing the feeding rate to the next higher charge state. In fact, approximately every third $\mathrm{Tl}^{+}$ion leaving REXTRAP ends up as a $\mathrm{Tl}^{53+}$ ion after the separator if charge bred for $390 \mathrm{~ms}$ in a $200 \mathrm{~mA}$ electron beam. This impressive performance underlines the holding capability inside REXEBIS, also for heavy ions and even when operated with a low-current electron beam.

The general trend of a decreasing relative abundance with $Z$ agrees with theory and becomes more distinct if the single charge state efficiency values are adjusted by dividing them by the EBIS global efficiency, as shown in Fig. 14 for a selection of elements. Thereby, the effect of injection and extraction losses, which were particularly penalizing the $\mathrm{Na}$ dataset in this measurement series, could be eliminated. In Fig. 14, the simulated relative abundances

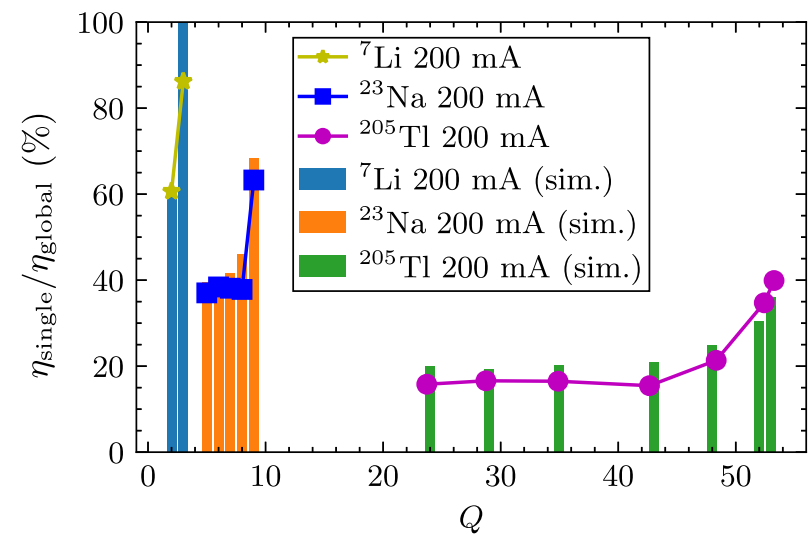

FIG. 14. For a selection of elements, the EBIS single charge state efficiency values have been divided by EBIS global efficiency. As a comparison to these curves, simulated relative abundances for the different charge states using the operational electron beam parameters are indicated as bars.

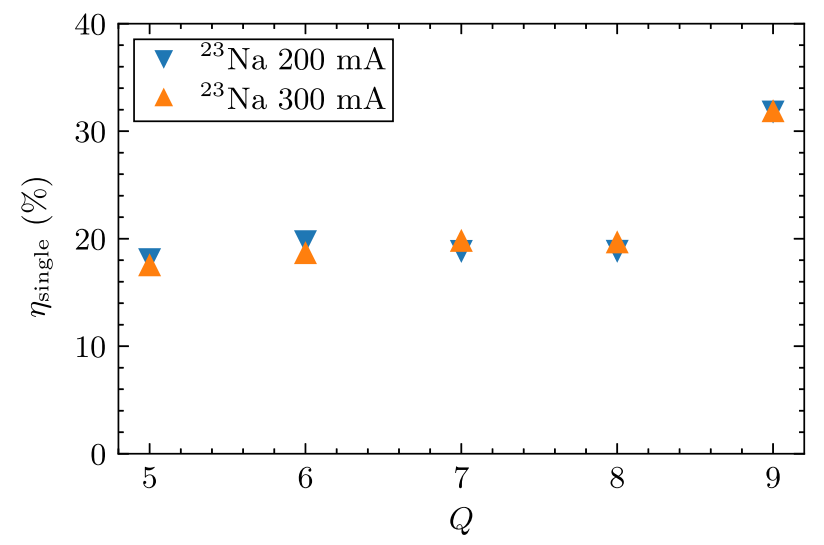

FIG. 15. EBIS single charge state efficiency versus charge state for $\mathrm{Na}$ charge bred with $200 \mathrm{~mA}$ and $300 \mathrm{~mA}$ electron beams.

are added under consideration of electron impact ionization and radiative recombination. The correspondence between measurement and simulation is excellent.

Similar to the global efficiency, the single charge state efficiency did not differ for the $200 \mathrm{~mA}$ and $300 \mathrm{~mA}$ electron beams, as illustrated in Fig. 15 for the two sets of $\mathrm{Na}$ data points. The significance of this is discussed in next section.

\section{Breeding efficiency discussion}

It is worth noting the insensitivity of the global efficiency to the electron beam current, as seen in Fig. 12. Since the $1+$ ion injection acceptance into a non-neutralized electron beam current with REXEBIS parameters is approximately proportional to the square root of the electron current [14], one would expect to observe a difference in efficiency, unless the $1+$ emittance of the injected beam is below the acceptance value. Earlier measurements at REXTRAP indicate emittances exceeding the acceptance [25,31].

In addition, the $1+$ ion injection energy inside the trapping region, measured with respect to the bottom of the electron beam well, is for optimal injection efficiency significantly higher than the actual potential well within the electron beam. The latter is $\approx 40 \mathrm{~V}$ for a $200 \mathrm{~mA}$ beam, while the injection energy varies with conditions between $200 \mathrm{eV}$ to $300 \mathrm{eV}$. An example of charge state distributions recorded for ${ }^{152} \mathrm{Sm}$ and $200 \mathrm{~mA}$ electron beam, while varying the injection energy from $250 \mathrm{eV}$ (nominal case) to $50 \mathrm{eV}$ and $450 \mathrm{eV}$, is given in Fig. 16. The efficiency for the two higher beam energies is similar, although for the third case, where the injection energy is similar to the potential well of the electron beam, a radical reduction in efficiency is apparent. If the injected beam emittance would fall within the predicted acceptance, one would expect no decline in injection efficiency even at the lower energy. The argument assumes that the beam tuning allows for matching between emittance and acceptance Twiss parameters. 


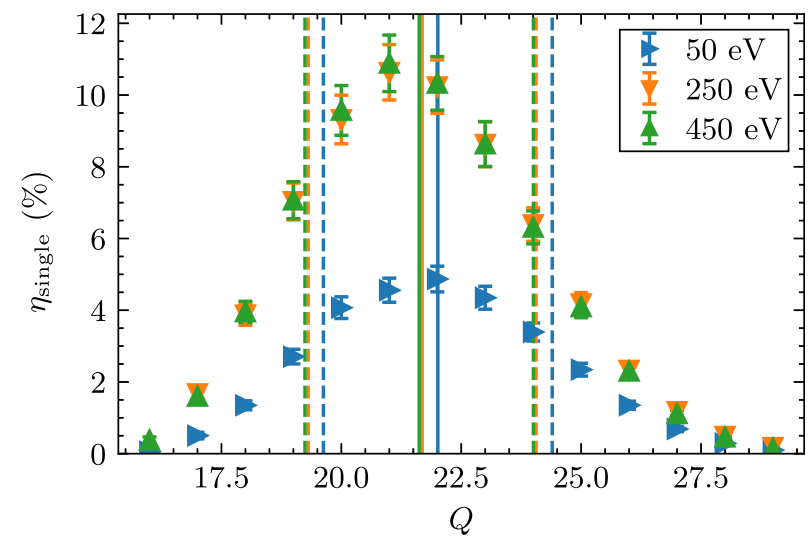

FIG. 16. EBIS single charge state efficiencies for ${ }^{152} \mathrm{Sm}$ injected into the EBIS trapping region with different beam energies (given in the legend). The vertical lines mark the mean and standard deviation of the charge state spectrum for the three injection energies.

Hence, we deduce that for a pulsed beam injection, the injected beam emittance may very well be larger than the predicted acceptance of the electron beam without affecting the efficiency, as long as the injection energy is sufficiently high for the ions to enter the trapping region. Of course, the injected bunch must propagate sufficiently slowly within the trap region to have time to be enclosed by the outer barrier when that is rapidly raised. In the REXEBIS case, the physical pulse length inside the trapping region is $9 \mathrm{~cm}$ and $11 \mathrm{~cm}$ for $A=6$ and 240, respectively, to be compared the trap length of $80 \mathrm{~cm}$. Here, an energy of $250 \mathrm{eV}$ was assumed, and measured pulse lengths out of the trap of $1 \mu \mathrm{s}$ and $8 \mu$ s (FWHM) were used.

With an injection energy appreciably larger than the potential well, one can imagine that recently injected ions may circle around the electron beam. From Fig. 16, there is no clear evidence of a shift in charge state distribution with injection energy. On the other hand, the distributions were probed after a $10 \mathrm{~ms}$ breeding time, at an average charge state of $21+$, and at this stage the ions will be completely trapped within the electron beam. In fact, with our operational parameters, above the charge state of approximately $10+$ all ions will be fully immersed inside the electron beam due to the augmented effective holding voltage with increased charge state. Nevertheless, from operational experience at REXEBIS, we know that the charge state distribution for light ions, and thereby low charge states, is to a certain degree affected by the injection conditions. This behavior has been reproduced for a $\mathrm{K}$ beam with the new gun and is presented in Sec. V D.

\section{Current density determination}

The electron beam current density $j$ in the ion trapping region is generally seen as one of the key figures of merit for an EBIS. Since the ionization rates are proportional to the current density, it has a direct impact on the breeding time that is required to reach the target charge state and determines the achievable repetition frequency of the source and linac operation.

During the commissioning tests the effective electron current density $j_{\text {eff }}$ has been determined. It relates to the actual current density as $j_{\text {eff }}=j f_{\text {ei }}$, where $0 \leq f_{\text {ei }} \leq 1$ is the so-called electron-ion overlap factor which describes the probability of finding a given ion within the confines of the electron beam. In general, the overlap factor, and hence the effective current density, may vary with ion species, charge state, temperature and the degree of compensation of the radial trapping potential due to the positive charge of the ions. In a radioactive ion beam charge breeder, where attained $A / Q$-values are usually moderate, such that Spitzer heating [32-34] has limited time to drive the ions out of the electron beam, the injection of the ions into the boundary of the electron beam can be of particular importance. A poor overlap of the injected ions with the electron beam can cause an initial staggering of the charge breeding process, leading to longer overall breeding times and more smeared out charge state distributions.

\section{Current density methodology}

To characterize the effective current density of the new electron gun, we have measured the charge state evolution in REXEBIS for various elements, with varying beam currents, and using external ion injection as well as neutral gas injection. The extracted ion beam was separated according to the $A / Q$-ratio with the spectrometer located downstream of REXEBIS and subsequently the current in a given charge state $Q$ was recorded using FC4 (see Fig. 9).

In order to extract the effective current density from the measurements, the measured charge state evolution has been fitted to the predictions of a simple theoretical model. As first demonstrated by Penetrante et al., the evolution of the charge state distribution inside an electron beam ion source can be modelled through a set of rate equations [35]. Here, we have chosen a simplified model excluding any thermal effects in order to limit the degrees of freedom and uncertainties in the simulation. Such a model provides a best-case scenario, since it is assumed that the overlap between electron and ion beam is perfect at all times, and that ions cannot escape from the trap. Furthermore, charge exchange has not been included due to its dependence on the ion temperature, and the low residual gas pressure in REXEBIS. As this model excludes numerous mechanisms inhibiting the charge breeding process, it should generally show a faster evolution than observed in practice. Therefore, the true current density is likely to be underestimated and the result should be interpreted as an effective density, presenting a lower limit for the true value.

In their simplified form, the rate equations are composed of terms for Electron Ionization (EI) and Radiative Recombination (RR) 
$\frac{d N_{Q}}{d t}=\frac{j_{\mathrm{eff}}}{e}\left(\sigma_{Q-1}^{\mathrm{EI}} N_{Q-1}+\sigma_{Q+1}^{\mathrm{RR}} N_{Q+1}-\sigma_{Q}^{\mathrm{EI}} N_{Q}-\sigma_{Q}^{\mathrm{RR}} N_{Q}\right)$.

Here, $N_{Q}$ denotes the number of ions in charge state $Q$ and $\sigma_{Q}^{\mathrm{EI} / \mathrm{RR}}$ are the associated cross sections for ionization and recombination. The cross sections have been computed according to the established Lotz formula for EI [36-38] and the Kim and Pratt formula for RR [39]. The required electron configurations and binding energies were taken from a set of existing tables $[19,40]$.

The absence of charge exchange in this model can be supported with a brief conservative estimation. During our current density measurements, the highest observed charge state was $\mathrm{Sm}^{49+}$. Considering charge exchange of these ions with Ne gas, the Müller and Salzborn formula [41] can be used to estimate a cross section of $2.8 \times 10^{-14} \mathrm{~cm}^{2}$. For ions moving with a thermal velocity of $1.27 \times 10^{6} \mathrm{~m} / \mathrm{s}$ (equivalent to a very high temperature of $10 \mathrm{keV}$ ) through a residual $\mathrm{Ne}$ gas of pressure $5 \times 10^{-11} \mathrm{mbar}$ at room temperature, the charge exchange rate is approximately $0.44 \mathrm{~s}^{-1}$. This can be compared to the ionization rate of $\mathrm{Sm}^{48+}$ ions in a beam with a current density of $200 \mathrm{~A} / \mathrm{cm}^{2}$. Using the Lotz formula the cross section for this process has been estimated to be $2.0 \times 10^{-21} \mathrm{~cm}^{2}$, resulting in an ionization rate of $2.5 \mathrm{~s}^{-1}$. This means that even in this extreme case, ionization outweighs charge exchange. Thanks to the favorable cross section scaling toward lower charged ions, charge exchange should be entirely insignificant for most of our data.

The fitting procedure can be described as follows: For a given dataset five geometrically spaced points in time are chosen as the support for a linear interpolator describing $j_{\text {eff }}(t)$. These points cover the time span between the shortest and longest measured charge breeding time for a given dataset, and the geometric spacing of the sampling times reflects the increasing characteristic timescales for higher charge states. This interpolator is inserted into Eq. (6) and the differential equation is solved numerically to simulate the charge breeding process. Using a standard least squares algorithm the values of $j_{\text {eff }}(t)$ at the chosen support points are adjusted until the best agreement between measurement and simulation is achieved. In addition to the five fit parameters describing the current density evolution there is a sixth one for the vertical scaling between data and simulation. As for the efficiency measurements in Sec. VC, the measurement error of the ion current was assumed to be $0.1 \mathrm{pA}+5 \%$ of the measured signal.

A Monte Carlo technique was chosen to estimate the uncertainty of the fitted current densities due to the lack of exact ionization cross sections. For this purpose, each fit was repeated 200 times while shuffling the ionization cross section in two ways. Firstly, the beam energy is assumed to be distributed uniformly in an interval of $\pm 100 \mathrm{eV}$ around the space charge corrected set value. For a uniform $200 \mathrm{~mA}$ $(300 \mathrm{~mA}), 6 \mathrm{keV}$ electron beam with a radius of $187 \mu \mathrm{m}$ inside a $5 \mathrm{~mm}$ radius drift tube, the total space charge depression is approximately $305 \mathrm{~V}(464 \mathrm{~V})$ where the intrabeam potential drop accounts for $40 \mathrm{~V}(61 \mathrm{~V})$. The uncertainty about the beam energy mostly stems from power supply calibration, and the lack of knowledge about the true beam profile and possible radial alignment issues which change the space charge deceleration of the beam. This is particularly important close to the ionization thresholds, where the cross section can vary quickly. Secondly, each individual ionization cross section was multiplied with uniformly distributed random factors between 0.7 and 1.3 to reflect the approximate nature of the Lotz formulae in a way that largely covers the magnitude of model errors reported by Lotz. This yields multiple fit results which were used to compute a mean and standard deviation for the current density value at each support point.

\section{Current density results}

The procedure described above has been used to determine effective current density values from charge breeding performance measurements for various elements and operating conditions. In the case of ${ }^{7} \mathrm{Li},{ }^{23} \mathrm{Na},{ }^{39} \mathrm{~K}$, and ${ }^{152} \mathrm{Sm}$ a beam of singly charged ions was injected into the EBIS; ${ }^{22} \mathrm{Ne}$ and ${ }^{129} \mathrm{Xe}$ were provided as neutral gases. The beam current was varied in the range $200 \mathrm{~mA}$ to $300 \mathrm{~mA}$.

Figure 17 shows an example of the current density fitting the charge breeding of ${ }^{23} \mathrm{Na}$ with an electron beam current of $300 \mathrm{~mA}$. The number of ions has been scaled by the period time of the operation cycle in order to account for the ion collection time in REXTRAP, and hence the total charge of the injected primary beam. The error bars of the data points reflect the assumed ion current error. The curves show the mean value of the simulated charge state evolution, whereas the surrounding bands reflect the model uncertainty determined with the Monte Carlo technique. The error bars of the fitted current densities are likewise a result of the Monte Carlo run.

The current density evolutions obtained from all measurements are summarized in Fig. 18 (top). It shows a wide range of effective current densities $\left(\approx 100 \mathrm{~A} / \mathrm{cm}^{2}\right.$ to $800 \mathrm{~A} / \mathrm{cm}^{2}$ ) for the different measurement scenarios. Some variability in these current densities can obviously be eliminated through normalization by the electron beam current. In this manner, the effective radii $r_{\text {eff }}=\sqrt{I_{e} / \pi j_{\text {eff }}}$ can be computed and are displayed in Fig. 18 (bottom). Presented in this way, the data points associated with the same element follow each other closely. However, clear differences remain between the ion species. The current density evolution generally shows a decreasing trend with increasing breeding time. Additionally, a correlation between the ion mass and the effective current density 

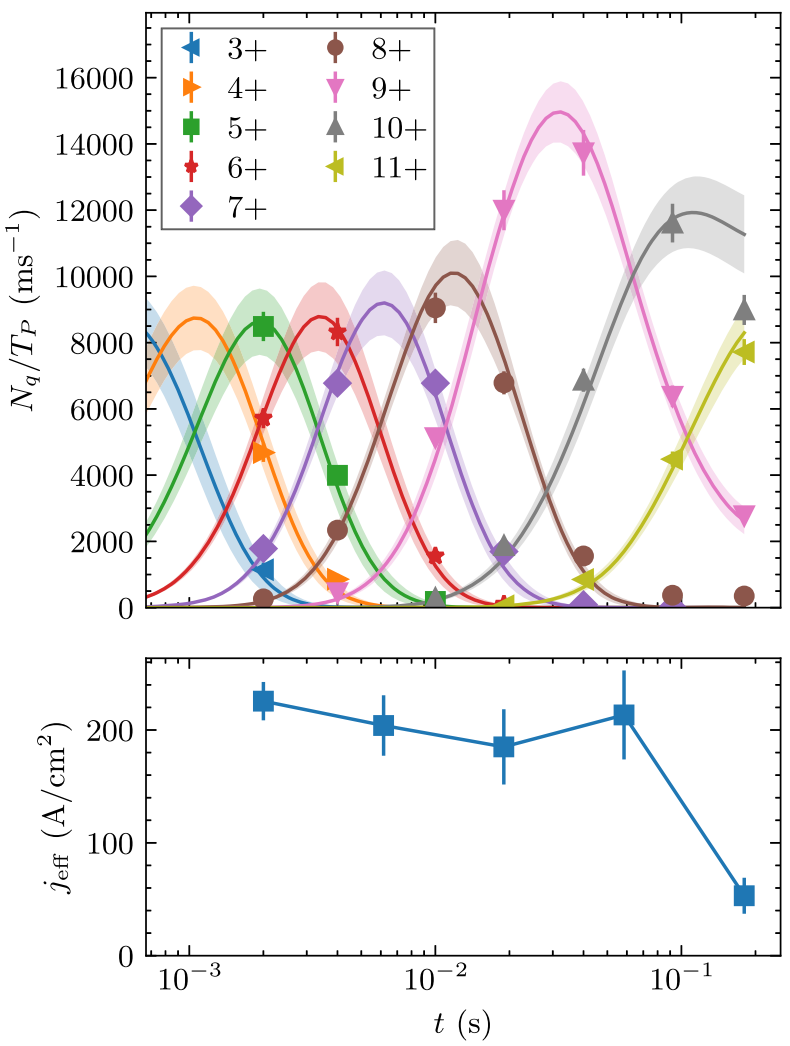

FIG. 17. Plot of the charge state evolution of ${ }^{23} \mathrm{Na}$ with a beam current of $300 \mathrm{~mA}$. The data points in the upper plot show the measured ion intensity (normalized to the primary beam accumulation time). The banded curves represent the evolution of the charge state distribution and its uncertainty according to the fitted theoretical model. The lower plot displays the fitted current density values at the sampling times. The uncertainty of the fitted parameters and the uncertainty bands of the theoretical evolution have been estimated with a Monte Carlo technique (see main text).

can be observed. This mass dependency is illustrated in Fig. 19 which shows a plot of the determined current densities as a function of the nuclear charge for the $200 \mathrm{~mA}$ electron beam measurements. The error bars show the sample standard deviation of the 5 individual current density values determining the time evolution. As such they represent the approximate dynamic range of the effective current density within a single breeding cycle.

Figure 20 contains the charge state evolution of ${ }^{39} \mathrm{~K}$ bred with a $200 \mathrm{~mA}$ electron beam. The measurements were carried out at particularly low charge states and short breeding times to capture the breeding behavior just after ion injection. The upper plot presents the measured charge state evolution for charge states $2+$ to $12+$. A striking feature of this measurement is the notable increase in amplitude for charge states $9+$ and higher. The lower plot shows a simulation for the charge breeding of $\mathrm{K}$ ions for an assumed current density of $200 \mathrm{~A} / \mathrm{cm}^{2}$.
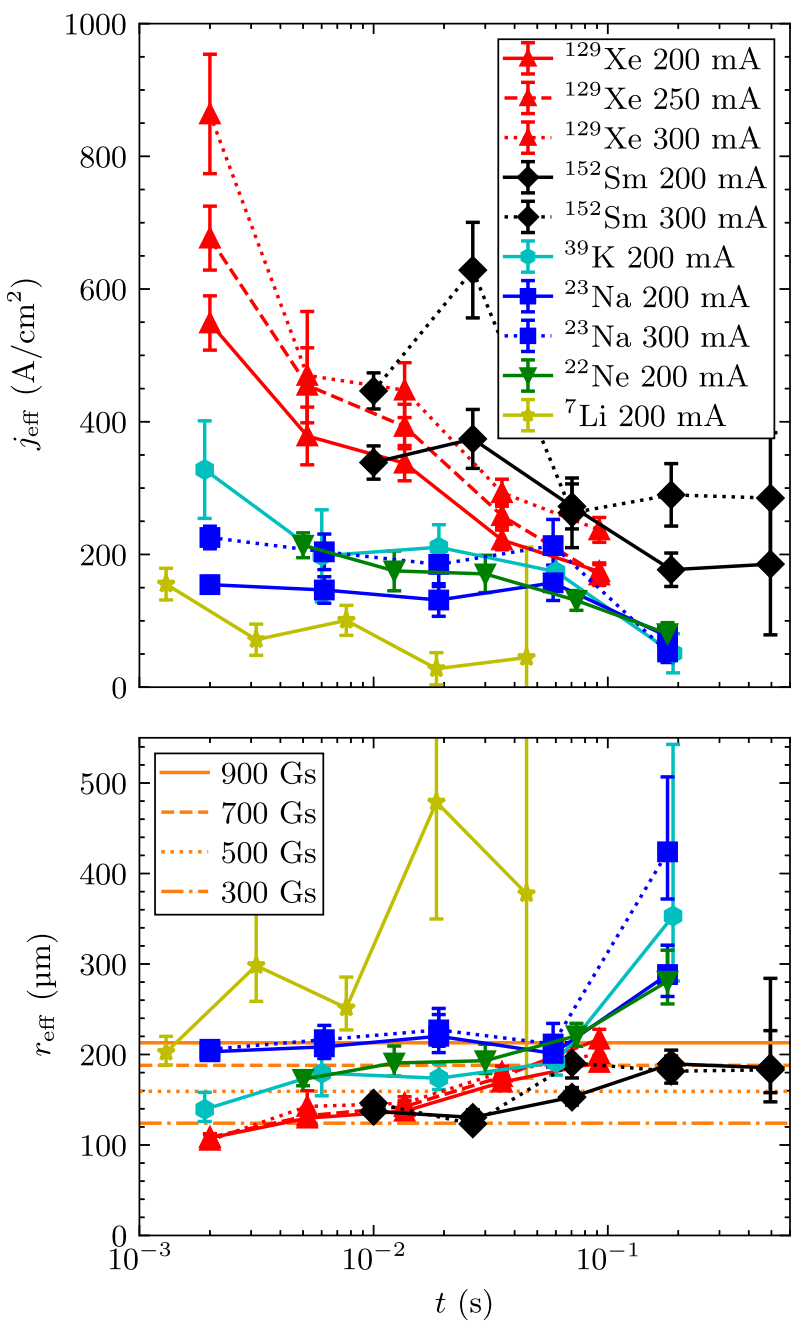

FIG. 18. Collective plot of the fitted current density values for all experiments. The upper plot contains the current densities as obtained from the fitting model. The lower plot shows the corresponding effective radii, obtained assuming a top hat density profile of the electron beam and ion cloud. The orange lines display the theoretical electron beam radii calculated for different magnetic field configurations at the cathode.

\section{Current density discussion}

The exemplary fitting results shown in Fig. 17 demonstrate that the chosen fitting model is generally able to reproduce the measured charge state distributions, despite the strong simplifications in the chosen rate equation (6). The agreement between the data and the fit within their respective error bars, provides confidence in the determined effective current densities.

However, the fitting results also clearly illustrate the difficulty of determining the true current density of the electron beam based on the evolution of the extracted ion distributions and the effective current densities fitted to the various datasets. The two most severe limitations to this method are firstly the lack of information about the overlap between the electron beam and the ion cloud, and secondly 


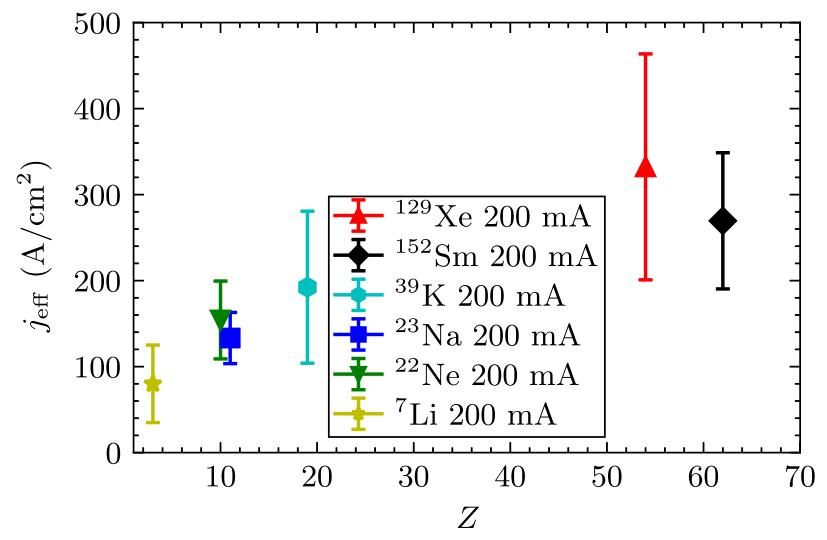

FIG. 19. Plot of the deduced electron current densities (at $200 \mathrm{~mA}$ electron current) for various elements versus the element number. The markers and error bars represent the arithmetic mean and standard deviation of the corresponding current density values shown in Fig. 18.

the large uncertainties associated with the ionization cross sections. For the cross sections themselves there are two sources of uncertainty, one being the shortfalls of the empirical cross section scaling models themselves, the second stemming from the uncertainty on the beam energy, which can imply significant cross section variations especially for cases where the beam energy is close to the
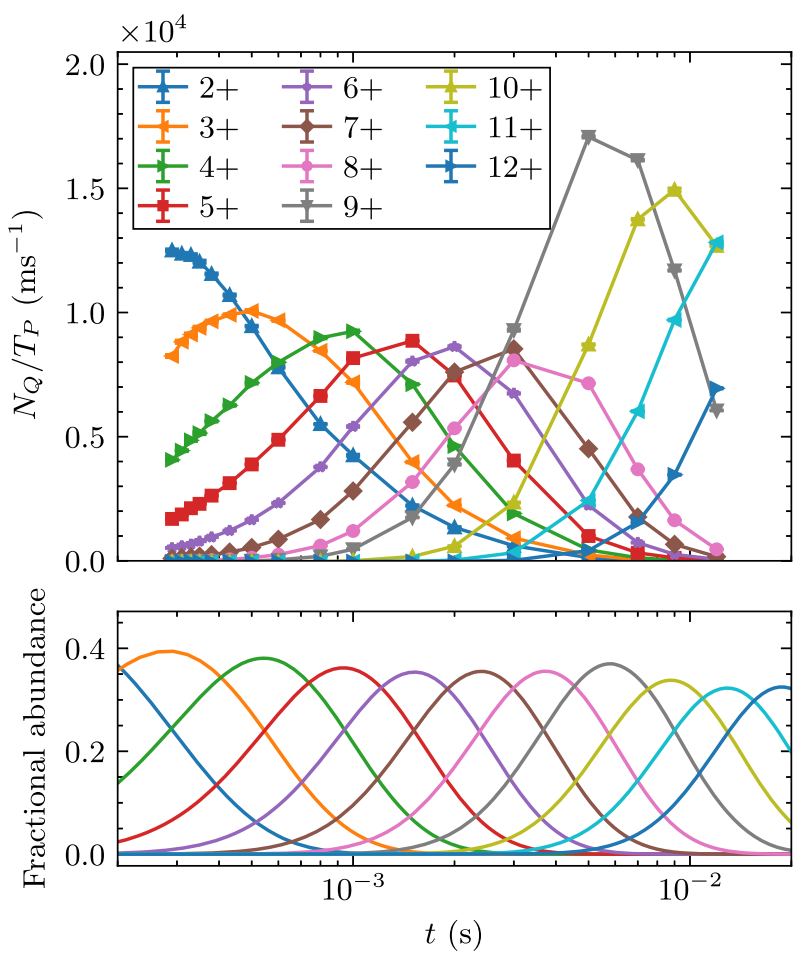

FIG. 20. Plot of the charge state evolution of ${ }^{39} \mathrm{~K}$ with a beam current of $200 \mathrm{~mA}$. The upper plot contains the measured ion output. The lower plot shows a simulation of the charge state evolution for an assumed current density of $200 \mathrm{~A} / \mathrm{cm}^{2}$. binding energies of the valence electrons. Whereas a diminishing overlap should generally cause an effective reduction of the current density, the ionization cross section uncertainties can affect the resulting current density in either direction.

The detailed charge state evolution of ${ }^{39} \mathrm{~K}$ in Fig. 20 provides evidence for both overlap effects and erroneous cross section estimations. Comparing the measurement to the simulation in the lower plot, it becomes apparent that the low charge state curves extend to significantly longer breeding times than expected from theory. This can likely be explained by a poor overlap between the injected $1+$ ions and the electron beam. This delays the first couple of ionization steps leading to a stretching of the curves. It can be thought of as a slow injection of $1+$ ions into the electron beam. The second notable difference between measurement and simulation is the amplitude jump for high charge states. This occurs at the $8+$ to $9+$ to $10+$ transition and is likely linked to shell closure effects for $\mathrm{K}^{9+}$. It appears that in this case the Lotz formula does not reproduce the ionization cross sections accurately enough to account for this significant difference in peak values. Yet, the fit of the Na data in Fig. 17 shows good results for the He-like shell closure, suggesting that not all shell closures are inherently problematic for the Lotz model. Before the submission of our manuscript we took notice of Ref. [42], where Angot et al. use the charge evolution of $\mathrm{K}$ tracer ions to determine the plasma parameters in an electron cyclotron resonance charge breeder. They report a similar efficiency increase at the $8+$ to $9+$ transition for ions bred with thermally distributed electrons.

In Fig. 18 (bottom), the effective electron beam radii are compared to the theoretical Herrmann radii of the electron beam for different magnetic flux densities on the cathode. The gun has been designed and installed aiming for a cathode flux density of $700 \mathrm{Gs}$, which should be seen as the baseline. Inaccuracies in the axial position of the gun may shift this value slightly, but deviations larger than \pm 50 Gs are not expected. Effective radii that are larger than the theoretical electron beam radius can be explained by an expansion of the ion cloud beyond the confines of the beam, since ionization is impeded for ions spending time outside the electron beam such that the effective current density is diminished. The reciprocal is not true, since the upper limit for the current density is given by the electron beam itself. One should therefore expect to see a minimum effective radius that agrees with the theoretical predictions.

The clustering of data points around the 500 Gs reference line can only partially be explained by an axial misalignment of the gun and an associated reduction of the cathode flux. It is more likely that the beam density profile is not uniform but rather bell-shaped. This can increase the local current density at the center of the beam above the average value associated with a uniform beam. If a given ion cloud is small enough to reside mostly within this high current 
density neighborhood, it would appear that the current density is higher than expected.

The mass trends displayed in Fig. 19 are largely in line with prior experience. Heavier and more highly charged ions are expected to have a better overlap with the electron beam and in the case of a bell-profile electron beam they can be confined to the very central region experiencing the peak current density. This trend is confirmed in our measurements. One can also see that for $\mathrm{Ne}$ and $\mathrm{Xe}$ the effective current densities are notably higher than those of ions with similar mass. We ascribe this to use of gas injection versus singly charged ion injection. When ions are created from neutral gas, they must do so inside the electron beam, providing a good overlap between ions and electron beam. Singly charged ions, on the other hand, may initially orbit the electron beam on trajectories that severely decrease the chance for collision with an electron before falling into the beam after one or more delayed ionization steps, if injected suboptimally. Additionally, the initial current density estimate for the Xe experiments may be inflated by the combination of continuous gas injection and short breeding times. If ions are created and charge bred prior to the nominal start of the breeding interval, they can distort the early charge state spectrum and raise the reconstructed current density. This is further supported by the observation of discrepancies between our measurement data and fits particularly for short Xe breeding cycles. For longer breeding times, this effect becomes insignificant as the contribution of early produced ions to the total signal is diminished.

While our measurements do not allow us to provide a precise value for the true current density, one may find it justified to assign an effective radius of approximately $150 \mu \mathrm{m}$ to $190 \mu \mathrm{m}$ to the electron beam, supported by the clustering of the reconstructed values in this regime. This corresponds to current densities of approximately $176 \mathrm{~A} / \mathrm{cm}^{2}$ to $283 \mathrm{~A} / \mathrm{cm}^{2}$ for the $200 \mathrm{~mA}$ beam, and $265 \mathrm{~A} / \mathrm{cm}^{2}$ to $424 \mathrm{~A} / \mathrm{cm}^{2}$ for the $300 \mathrm{~mA}$ case. These values are notably higher than the simulation based predictions in Sec. II B. Ultimately, the charge breeding performance of the EBIS depends on the electron beam, the mass and nuclear charge of the injected ion, and the injection conditions.

\section{E. Axial energy distribution measurements}

The underlying objectives for probing the axial energy distributions are to determine ionic temperatures and ultimately to evaluate the overlap between the ion density distribution and the electron beam. Cold ions orbit close to the electron beam axis and reside deep in the space charge potential. They are ionized at a faster rate than hotter ions which may eventually escape. The energy balance of ions in an EBIS is mainly affected by Coulomb heating (or Spitzer heating), ionization heating when the ions increase their charge state, and evaporative cooling. Quantifying the ionic temperature gives additional insight into these phenomena.

\section{Axial energy methodology}

In the context of slow extraction [10], a method has previously been implemented at REXEBIS to retrieve the axial energy distribution of the trapped ions. The technique for probing the axial energy distribution consists of progressively reducing the outer trapping barrier of the EBIS in consecutive spills and capturing only the corresponding ions of interest, after $A / Q$-separation. During the normal ion extraction scheme, the outer barrier potential is dropped from $1.3 \mathrm{kV}$ to platform potential, producing a pulse of ions with a time structure shorter than $100 \mu$ s. For the axial energy scan, however, at each step the outer barrier is first set to an intermediate probing potential for $0.5 \mathrm{~ms}$, at which the ion escape is recorded using the Faraday cup FC4 (Fig. 9), while ions residing at a lower potential are still trapped and only ejected after another millisecond when the whole trap is emptied as a preparation for the next breeding cycle. The timing of FC4 is set to only record the ions being extracted at the intermediate potential, so that the integral of the axial energy distribution can be reconstructed. It is worth mentioning that the $\operatorname{REX} A / Q$-separator has a sufficiently large energy acceptance to ensure full transmission efficiency even when the ions are extracted at nonnominal EBIS potentials. An equivalent procedure involves lifting the trapping potential rather than lowering the outer trapping barrier, and both methods have yielded similar results in the past.

In order to characterize the recorded energy distributions, a theoretical energy distribution is fitted to the measurement. The energy distribution of particles following MaxwellBoltzmann statistics, is described by a $\operatorname{Gamma}(k=$ $\left.n_{D} / 2, \theta=k_{B} T\right)$ distribution, where $n_{D}$ is the number of degrees of freedom. We have opted to present the fit results for a distribution with $n_{D}=3$ degrees of freedom (3D), due to the clearly superior goodness of fit compared to $n_{D}=1$. A brief refutation for choosing $n_{D}>3$ follows in Sec. VE 3 .

We present the escape current $I_{\mathrm{esc}}$ as a function of the intermediate barrier voltage $U_{B}$, such that the data follows the survival function $S$ [43]

$$
\begin{aligned}
I_{\mathrm{esc}} & =C \cdot S_{\mathrm{Gamma}\left(3 / 2, k_{B} T\right)}(E) \\
& =C\left[1-\frac{1}{\Gamma(3 / 2)} \gamma\left(3 / 2, \frac{E}{k_{B} T}\right)\right],
\end{aligned}
$$

where,

$$
\begin{aligned}
E & =q\left(U_{B}-700 \mathrm{~V}\right)-E_{0} \\
& =q\left(U_{B}-700 \mathrm{~V}-U_{0}\right) .
\end{aligned}
$$

Here, $C$ describes the signal amplitude, whereas $\Gamma$ and $\gamma$ denote the conventional and the incomplete gamma functions, respectively. The particle energy $E$ is determined by 
its charge $q$ as well as the barrier voltage relative to the trap potential of $700 \mathrm{~V}$, set for all measurements. Additionally, $E_{0}$ or $U_{0}$ can cover a shift of the energy distribution which may occur for example due to an offset of the applied potentials. This model was fitted to the experimental data using a conventional least squares method, yielding values for $k_{B} T, C$, and $E_{0}$.

In order to interpret and compare the temperature values of different measurements, it proves useful to normalize the determined temperature and relate it to the radial space charge well of the electron beam as $k_{B} T /\left(q \Phi_{0}\right)$. Here, $\Phi_{0}$ was chosen as the potential drop within the electron beam. For a uniform beam profile this potential well is independent of the beam radius to first order and can be calculated as

$$
\Phi_{0}=\frac{I_{e}}{4 \pi \epsilon_{0} v_{e}}
$$

The electron velocity $v_{e}$ can simply be determined from the electron beam energy, including an iterative correction for the space charge retardation.

\section{Axial energy results}

Axial energy distributions have been recorded for numerous elements, charge states, and breeding times, such that we cannot show the raw data for all measurements. Instead, the results are presented here in a condensed form.

Figure 21 shows three typical axial energy scans, and the corresponding 3D Maxwell-Boltzmann curves that were fitted to the data according to the procedure described in Sec. V E 1. In the plot, both the data and the fit have already been normalized by the fitted amplitude, to ease the visual presentation. The amplitude only represents the full beam current in a given charge state and is not of major

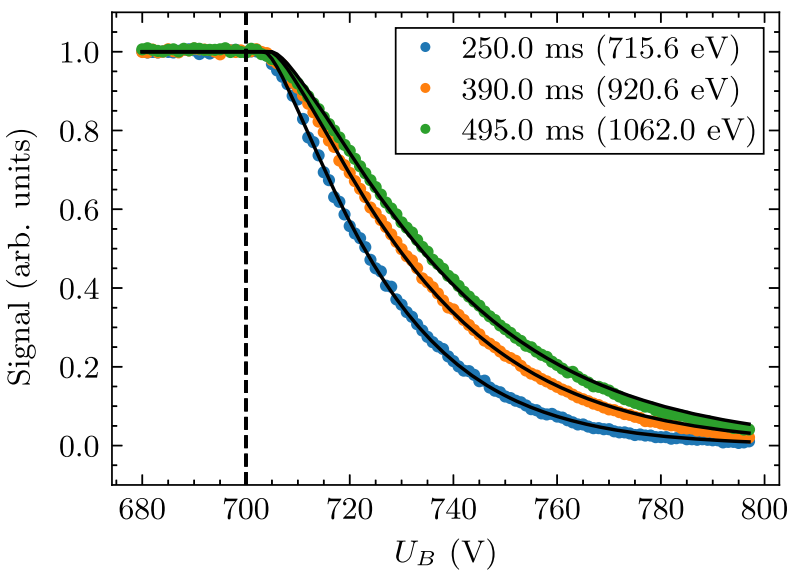

FIG. 21. Examples of axial energy measurements for ${ }^{152} \mathrm{Sm}^{44+}$ recorded at a beam current of $300 \mathrm{~mA}$. The data is overlaid with fitted energy distributions (see main text for explanation). The breeding times and fitted temperatures for the three cases are given in the legend. importance for the energy studies. It is rather connected to the charge state evolution and the determination of the effective current density from the axial energy scans.

The fitting parameters $E_{0}$ and $k_{B} T$ are those that hold information about the energy distribution. Figure 22 shows how these parameters vary with breeding time and charge state for the example of ${ }^{152} \mathrm{Sm}$ being charge bred with a $300 \mathrm{~mA}$ electron beam. The temperature generally increases over time and this trend has consistently been observed in all datasets. Furthermore, we have not observed major temperature discrepancies between neighboring charge states for the same breeding time. Here, the temperature is presented, normalized by the charge state, such that the resulting value can be interpreted as an approximate holding voltage needed to confine these ions. It was found to be $\leq 40 \mathrm{~V}$ across all measurements. While $E_{0}$ increases over time, normalization by the charge state reveals that $U_{0}=E_{0} / q$ generally fluctuates between $3 \mathrm{~V}$ to $6 \mathrm{~V}$. The only clear exception to this is the scan for ${ }^{23} \mathrm{Na}$, cf. Fig. 23, where $U_{0}$ falls from approximately $20 \mathrm{~V}$ for $6+$ ions at $2 \mathrm{~ms}$ breeding time to the generally observed range of $3 \mathrm{~V}$ to $6 \mathrm{~V}$ after approximately $20 \mathrm{~ms}$ breeding time.

The temperature evolution for all measurements is shown in Fig. 24. The curves illustrate the average behavior for a given measurement series. In cases where more than one charge state has been measured for a particular breeding time, the error bars indicate the sample standard deviation. Since we did not encounter large temperature
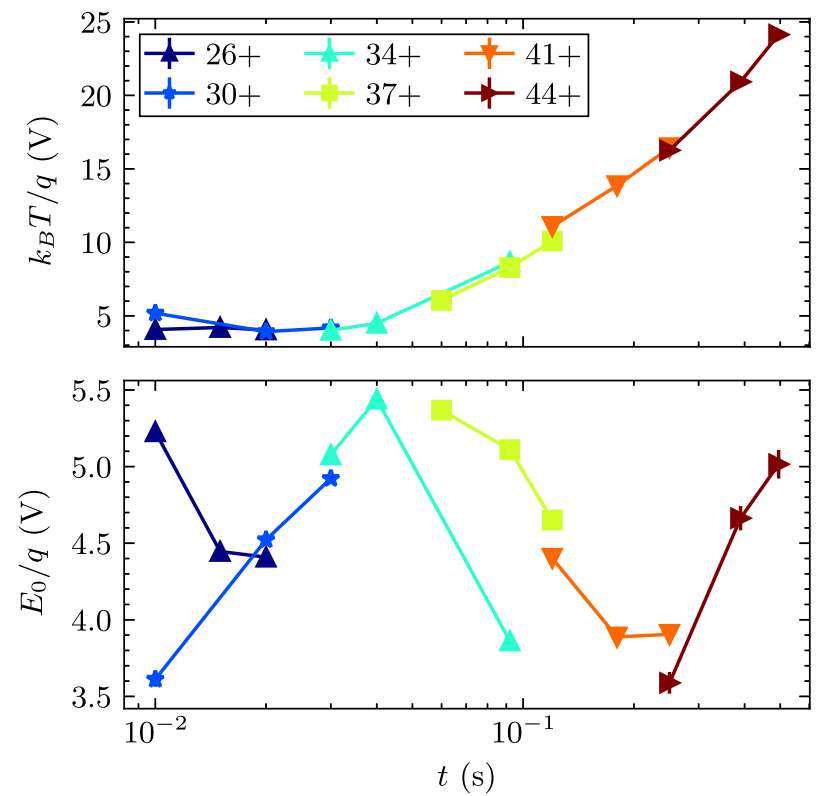

FIG. 22. Plot of the axial energy spectrum fit parameters for ${ }^{152} \mathrm{Sm}$ (300 mA beam current). Both the temperature (upper graph) and the axial energy offset (lower graph) have been normalized by the ion charge under investigation (indicated in the legend) to yield equivalent voltages. 

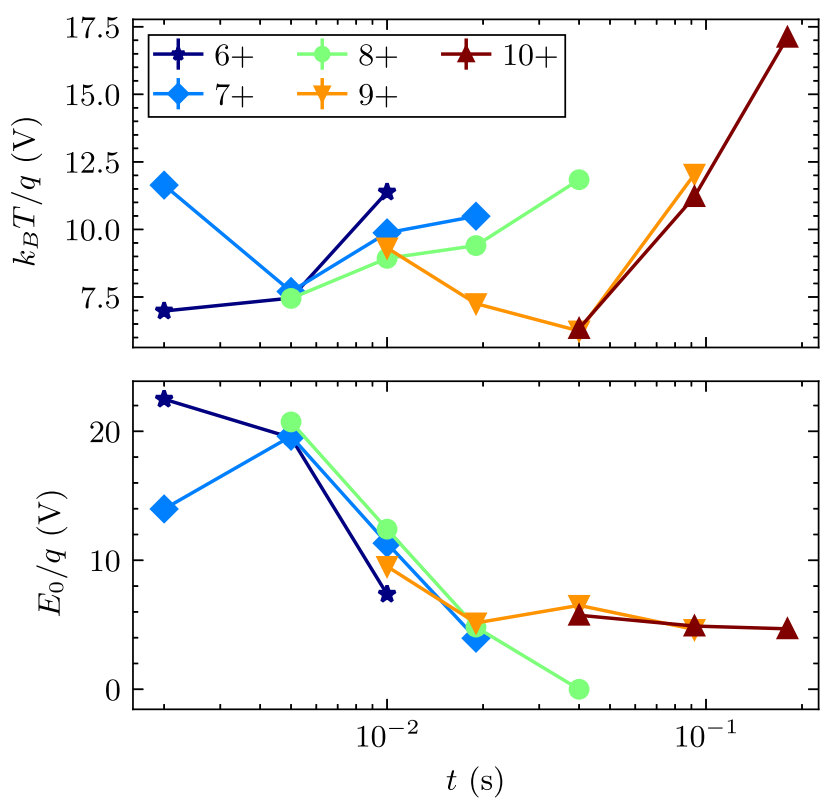

FIG. 23. Plot of the axial energy spectrum fit parameters for ${ }^{23} \mathrm{Na}$ (200 mA beam current). See Fig. 22 for further explanation.

discrepancies between charge states for the same breeding time, these error bars are generally small.

The upper plot in Fig. 24 illustrates the temperature evolution of ${ }^{152} \mathrm{Sm}$ for beam currents of $200 \mathrm{~mA}$ and $300 \mathrm{~mA}$. Moreover, there are two traces for the charge breeding of ${ }^{23} \mathrm{Na}$, where one trace illustrates a measurement, in which a Xe injection valve had accidentally been kept open. The enhanced heating of ${ }^{23} \mathrm{Na}$ in this case, compared to the case without Xe injection is clearly visible. In addition, the ${ }^{22} \mathrm{Ne}$ dataset presents a temperature evolution in which ions were created from residual gas as opposed to external ion injection as in the other cases.

After normalization by charge state and the characteristic trapping potential, cf. Fig. 24 (bottom), the curves for ${ }^{152} \mathrm{Sm}$ fall closely together regardless of the electron beam current. For the significantly lighter elements ${ }^{22} \mathrm{Ne}$ and ${ }^{23} \mathrm{Na}$, the normalized temperature is generally higher than for $\mathrm{Sm}$. While the (uncontaminated) $\mathrm{Na}$ and $\mathrm{Ne}$ curves start out at different values, they follow each other more closely after approximately $40 \mathrm{~ms}$.

\section{Axial energy discussion}

The increasing temperature observed in Fig. 24 agrees with the notion that various processes like Spitzer heating and ionization heating drive up the ion temperature. The more time they have got to act, the higher the temperature will be. Due to thermal coupling of many ion species in the trap, solid theoretical predictions for the heating rate are difficult. As a sanity check we have estimated the Spitzer heating rate $[32,34]$ for the ions investigated in our temperature studies
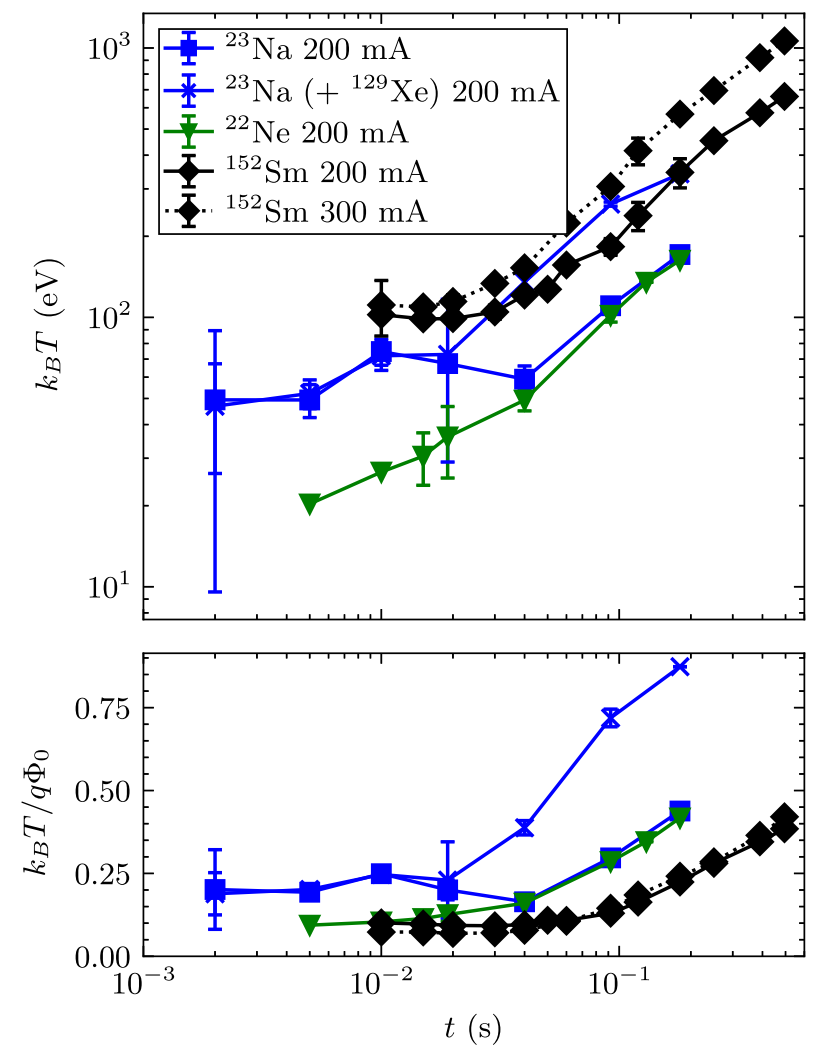

FIG. 24. Comparison plot of the temperature evolutions in various charge breeding scenarios (top). The element and electron beam current are indicated in the legend. Every data point represents several charge states; the error bars show the standard deviation of the temperature across all sampled charge states at a given breeding time. In the lower plot the temperature is rescaled with the charge state and the depth of the space charge potential well within the electron beam.

$$
\left[\frac{d\left(k_{B} T\right)}{d t}\right]^{\mathrm{SH}}=\frac{2}{3} n_{e} v_{e} 4 \pi\left(\frac{Q e^{2}}{4 \pi \epsilon_{0} m_{e}}\right)^{2} \frac{\log \Lambda_{\mathrm{ei}}}{v_{e}^{4}}\left(2 \frac{m_{e}}{m_{i}} E_{e}\right) .
$$

Here, $n_{e}$ is the electron density in the beam, $E_{e}$ denotes the electron beam energy, $m_{e}$ and $m_{i}$ are the electron- and ion mass, respectively, and $\log \Lambda_{\mathrm{ei}}$ is the electron-ion Coulomb logarithm.

For Sm, the observed heating rates were found to be smaller than the Spitzer heating rates, by roughly $0 \%$ to $80 \%$. As Sm is likely to be cooled by the lighter ions in the trap, this discrepancy is expected. For $\mathrm{Ne}$ and $\mathrm{Na}$, the observed heating rates did occasionally exceed the Spitzer estimate, but generally not by more than $300 \%$. As these are light and lowly charged ions, they are more susceptible to the effects of ionization heating and ion-ion heating. Considering these supplementary effects, the measurement results are not in complete disagreement with theoretical predictions. 
During the charge breeding interval, the axial barrier is kept at a voltage of $600 \mathrm{~V}$ above the central trap potential and the depth of the space charge well formed by the electron beam typically exceeds $300 \mathrm{~V}$. Since the equivalent holding voltage $k_{B} T / q$ did not exceed $40 \mathrm{~V}$ in any of our measurements, one can speak of a deep trap, and hence ion escape and evaporative cooling should largely be suppressed. This agrees with the observed high survival rate of ions during the charge breeding independent of the confinement time as presented in Sec. V C 3 .

The observed fluctuation of $U_{0}$ in the range of $3 \mathrm{~V}$ to $6 \mathrm{~V}$ can probably be explained as due to small voltage offsets in the analog-controlled power supplies feeding the drift tubes, resulting in disagreement between the assumed and actual trap depth. It should be mentioned that we also used the observed evolution of $U_{0}$ to discard our attempts of fitting the thermal distribution with 5 degrees of freedom. The notion that the ions may follow a distribution with 5 degrees of freedom due to their spread in the radial trapping potential was brought forward by Currell et al. in Refs. [34,44]. Since the energy distribution is dominated by the exponentially dropping high energy tail, no strong case could be made for either 3 or 5 degrees of freedom based on the goodness of fit alone. For 5 degrees of freedom however, $U_{0}$ showed a consistent decrease over time. This behavior cannot be explained by the trap dynamics. If the trap were compensated significantly, such that the effective trap potential is increasing, then $U_{0}$ should increase over time. However, there is no obvious mechanism that would drive $U_{0}$ in the opposite direction. We wish to underline that we are not making a statement about the actual distribution inside the trap, but we find a 3D distribution to best describe the axially escaping ions. It is well possible that these are just 3 degrees of freedom that contribute particularly well to axial escape on sub millisecond timescales, whereas additional degrees of freedom may still exist inside the trap.

As mentioned, the ${ }^{23} \mathrm{Na}$ measurement was found to display a strongly decreasing trend of $E_{0}$ for the initial $20 \mathrm{~ms}$. We interpret this as an excess in kinetic energy left over from the ion injection procedure. Singly charged ions are typically injected with an excess energy of $200 \mathrm{eV}$ to $300 \mathrm{eV}$. For a dilute ion cloud the collision rate between ions is relatively small, such that this energy is not instantly redistributed to follow a thermal distribution. Given that excess ion energy can be linked to a poor overlap with the electron beam, this agrees with the observed ionization delay for low K charge states in Fig. 20.

The injection energy can also explain the differences in the temperature evolution of ${ }^{22} \mathrm{Ne}$ and ${ }^{23} \mathrm{Na}$. While these ions are very similar in mass and charge, a higher temperature and energy offset are observed for the externally injected $\mathrm{Na}$ ions, compared to the gas injected Ne. Only after approximately $40 \mathrm{~ms}$, once the $\mathrm{Na}$ ions have been largely thermalized and their injection energy becomes insignificant compared to the total energy, do the temperature evolution of $\mathrm{Na}$ and $\mathrm{Ne}$ follow each other closely. Last, but not least, the curve for ${ }^{23} \mathrm{Na}$ with additional Xe injection illustrates how the presence of heavy ions, which are affected more strongly by Spitzer heating, can drive up the temperature of lighter ions through collisional heat transfer.

One might expect that the values shown in the lower plot of Fig. 24 correlate with the overlap between the electron beam and the ion cloud. The displayed values relate the ion temperature to the trapping potential they experience within the electron beam. If ions can move freely in the radial direction, their density profile should follow a Boltzmann distribution $\sim \exp \left(q \Phi / k_{B} T\right)$ after thermalization. Hence, a low normalized temperature should correspond to a more tightly radially confined ion cloud than a high value. In practice, however, it is difficult to find a consistent relationship between the temperature measurements, and the current density evolution in Sec. V D. Qualitatively, the increase of the temperature values obtained from the Maxwell-Boltzmann fits is consistent with the observed decrease in effective current density as ions are confined for longer breeding times. Furthermore, Fig. 24 (bottom) suggests a better confinement of Sm compared to the lighter elements for intermediate and long breeding times. Yet, we were unable to identify an electron beam model that, paired with Boltzmann distributed ions, could translate the measured temperatures into effective current densities which correspond to those determined from the charge state evolution. The problem is underlined by the initially very similar normalized temperatures for $\mathrm{Ne}$ and $\mathrm{Sm}$. This suggests that they should experience a similar overlap and hence similar current densities, in contrast to our results in Fig. 18, regardless of the assumed radial electron beam profile.

\section{F. Vacuum and beam contamination}

\section{Motivation and measurement techniques}

The analysis of $A / Q$-scans allows the deduction of partial pressures of residual gases inside the trapping region. The charge breeding performance is optimal with a low compensation of the electron beam but benefit from the presence of a low-mass background gas for the cooling of higher-mass ions. The presented $A / Q$-scans of the abundant residual gas were performed by scanning the separator magnet after REXEBIS while recording the average current on Faraday cup FC4. The connected picoamp meter has a resolution of 10fA. With a horizontal slit of $3 \mathrm{~mm}$-opening inserted at the focal point of the $\mathrm{A} / \mathrm{Q}$-separator, the resolving power is close to $\Delta_{A / Q} /(A / Q)=1 / 300$.

The composition of the ionized residual gas can also potentially contain cathode material. Therefore, the intensities of Ir and Ce in the extracted beam were probed, in order to verify that the contamination by the elements 
coming from the cathode does not considerably affect the purity of the beam. In $A / Q$-regions with weaker intensities, the separated beam was accelerated to $300 \mathrm{keV} / \mathrm{u}$ in the RFQ cavity and subsequently recorded on a silicon detector. The Passivated Implanted Planar Silicon (PIPS) detector used is $300 \mu \mathrm{m}$ thick and with a large diameter of $28 \mathrm{~mm}$ to assure a complete beam capture. By means of an energy histogram, the individual masses of the elements contained in the beam at a certain $A / Q$ could be determined. To avoid detector pile-up, calibrated attenuation grids were used yielding close to single-particle impact during each extracted pulse. The results shown below have been corrected for the attenuation factor. The method is described further in Ref. [45].

\section{Neutralization time and estimated partial pressures}

The extracted charge and electron beam neutralization as a function of breeding time was studied for a $202 \mathrm{~mA}$, $6200 \mathrm{eV}$ electron beam. In the first case, the valve to REXTRAP was closed, thereby preventing $\mathrm{Ne}$ from streaming into the EBIS, while with the open valve and the gas inlet into the Penning trap set at nominal value, $\mathrm{Ne}$ gas could enter the electron beam. The results are shown in Fig. 25. For a long breeding time of $300 \mathrm{~ms}$, a total charge of $\approx 570 \mathrm{pC}$ is extracted per pulse, corresponding to a neutralization factor of $17 \%$. The neutralization factor for the same breeding time, but with the valve closed is $<6 \%$, making Ne from the Penning trap the main contributor to the ionic space charge. As the residual gas data was acquired early in the commissioning campaign, the residual gas pressure is assumed to have been lower during most of the remaining efficiency, current density and axial energy scan measurements.

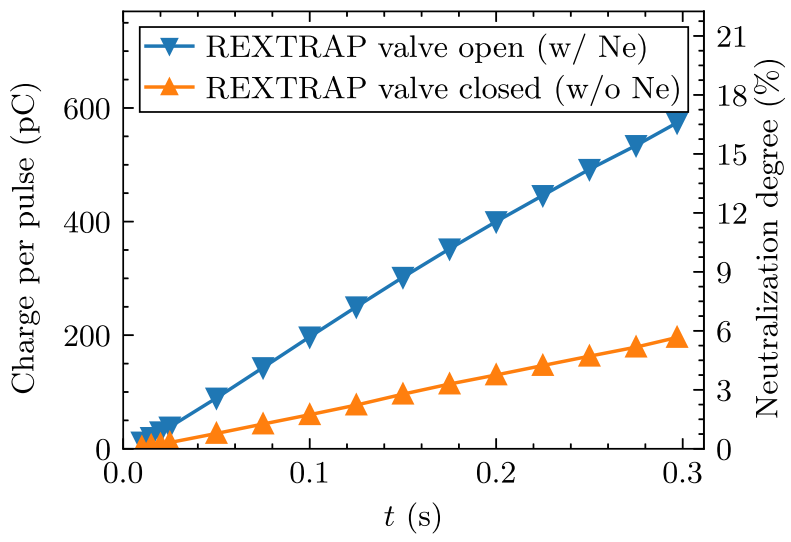

FIG. 25. Neutralization and extracted charge per pulse as a function of charge breeding time for a $202 \mathrm{~mA}, 6200 \mathrm{eV}$ electron beam. The recorded pressures in the gun and collector vacuum crosses were $1.2 \times 10^{-10} \mathrm{mbar} / 1.2 \times 10^{-10} \mathrm{mbar}$ and $7.2 \times 10^{-10} \mathrm{mbar} / 7.7 \times 10^{-10} \mathrm{mbar}$, respectively, with the valve to REXTRAP being closed/open.
The characterization of the ionized residual gas during charge breeding is crucial for estimating the quality of the vacuum inside the trapping region, and furthermore, to anticipate the purity of the beam of interest. The deduction of the individual atomic partial pressures is obtained in a semiempirical manner by comparing the measured residual gas spectra from REXEBIS to synthetic charge state distributions in close analogy to the method presented in Sec. VD 1. The spectra of ionized residual gas emitted from REXEBIS for a $200 \mathrm{~mA}$ electron beam were recorded repeatedly for different breeding times between $5 \mathrm{~ms}$ to $50 \mathrm{~ms}$. Figure 26 displays typical examples of the massscans obtained for a set of two different breeding times and with the valve to REXTRAP closed, implying that no Ne gas was injected.

Certain charge states are superpositions of different species, for instance, ${ }^{20} \mathrm{Ne}^{\mathrm{Q}+}$ with ${ }^{40} \mathrm{Ar}^{2 \mathrm{Q}+}$. Here, the fitting of artificial charge state spectra can help to disentangle the measured charge state distributions. At least three relevant
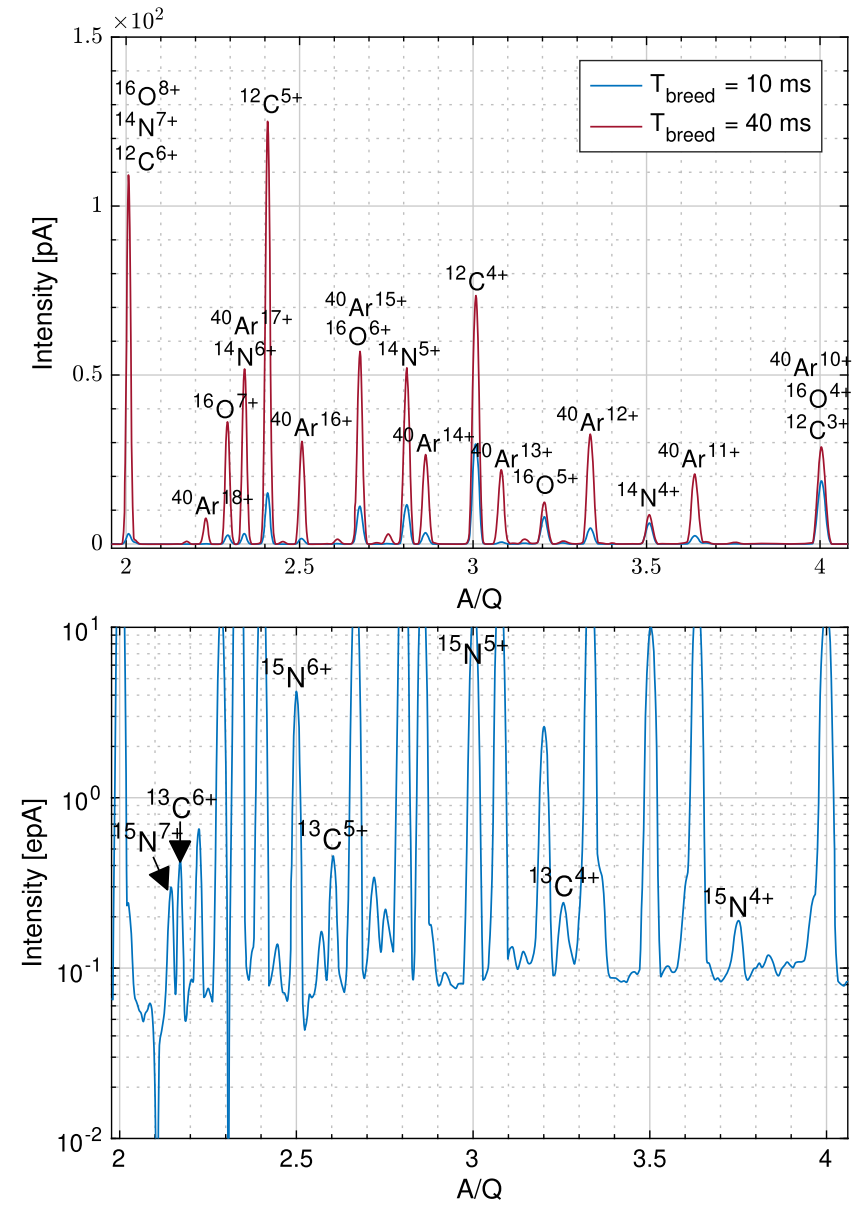

FIG. 26. The upper figure shows mass-scans acquired using a Faraday cup while sweeping the magnetic field of the $A / Q$ separator. Isotopes with relative abundances less than or equal to $1 \%$ are not labeled. The lower figure shows the dataset for $T_{\text {breed }}=10 \mathrm{~ms}$ with a logarithmic scale and identification of ${ }^{13} \mathrm{C}$ (abundance $1.07 \%)$ and ${ }^{15} \mathrm{~N}(0.36 \%)$. 
clean charge states are available for each residual gas species, and are used to deduce the corresponding effective electron beam current density $j_{\text {eff. }}$. This is achieved by fitting artificial spectra generated by the model presented in Sec. VD 1 to the measured spectra. In this context, the procedure was simplified by assuming a single constant current density, and skipping the Monte Carlo technique. The effective current density for the charge breeding of residual gas is found to be $j_{\text {eff }}=180 \mathrm{~A} / \mathrm{cm}^{2}$ for a $200 \mathrm{~mA}$ electron beam current, yielding an electron beam radius in perfect agreement with the Herrmann prediction. The second step is to determine the rate of neutral particles $R_{0}$ constantly injected into the electron beam. For this purpose, the measured beam intensities of the available charge states are converted into an equivalent number of particles $N_{Q}$, and the simulations are fitted to match the observed absolute numbers during charge breeding. The fitting model allows a reconstruction of the complete evolution of the total number of particles for all the charge states, $\sum_{Q} N_{Q}$. This term follows a linear increase as a function of time, which corresponds to the constant rate of neutral particles feeding the electron beam. Once the rate $R_{0}$ is known, the partial pressure of each species is derived according to the following formula,

$$
R_{0}=\frac{d}{d t}\left(\sum_{Q} N_{Q}\right)=p_{0} \frac{I_{e}}{e} \frac{L_{\text {trap }}}{k_{B} T_{0}} \sigma_{0}^{\mathrm{EI}}
$$

The main hypothesis behind the use of this formula is the ideal gas law to determine the neutral gas pressure $p_{0}$. However, it should be noted, that many of these ions are expected to originate from diatomic and organic molecules, such that the values provided here should not be interpreted as true pressures, but equivalent monoatomic pressures. For the calculation of the partial pressures, we assume a temperature $T_{0}=300 \mathrm{~K}$. A summary of the partial pressures in the trapping region and the corresponding constant number of neutral particles $N_{0}\left(r \leq r_{\text {beam }}\right)$ inside the electron beam volume with an assumed radius of $r_{\text {beam }}=$ $188 \mu \mathrm{m}$ is presented in Table I. To evaluate the partial pressure of $\mathrm{Ne}$, similar spectra to the ones presented in Fig. 26 were acquired, this time with the valve leading to REXTRAP opened and the buffer gas being injected. The partial pressures in the trapping region are significantly lower than in the neighboring gun and collector crosses, due to the high pumping speed of the NEG strips surrounding the drift tube structure.

\section{Residual mass spectrum and beam contamination}

The presence of evaporated Ce and Ir from the cathode surface was investigated by implanting the extracted and accelerated beam onto a silicon detector. Approximate charge breeding times were calculated such that the most abundant isotopes $\left({ }^{140} \mathrm{Ce}\right.$ and $\left.{ }^{193} \mathrm{Ir}\right)$ would end up in the
TABLE I. The atomic partial pressures of the main residual gas species ionized in REXEBIS.

\begin{tabular}{lcc}
\hline \hline Element & Pressure $(\mathrm{mbar})$ & $N_{0}\left(r \leq r_{\text {beam }}\right)$ \\
\hline $\mathrm{H}$ & $3.9 \times 10^{-11}$ & $8.4 \times 10^{4}$ \\
$\mathrm{C}$ & $2.9 \times 10^{-12}$ & $6.2 \times 10^{3}$ \\
$\mathrm{~N}$ & $5.2 \times 10^{-12}$ & $1.1 \times 10^{4}$ \\
$\mathrm{O}$ & $8.7 \times 10^{-13}$ & $1.9 \times 10^{3}$ \\
$\mathrm{Ne}$ & $1.9 \times 10^{-11}$ & $4.1 \times 10^{4}$ \\
$\mathrm{Ar}$ & $1.2 \times 10^{-12}$ & $2.5 \times 10^{3}$ \\
Total & $6.8 \times 10^{-11}$ & $1.5 \times 10^{5}$ \\
\hline \hline
\end{tabular}

$A / Q \approx 4.15$ region, which is relatively clean from other contaminating elements. The identification of the isotopes was verified with several neighbor charge states of Ir, Ce and Xe. To reach the necessary electron beam current of $300 \mathrm{~mA}$, the cathode temperature was set to $\approx 1900 \mathrm{~K}$. The result is displayed in Fig. 27, and despite the poor energy resolution of the silicon detector, one can see that the contributions to the beam from both $\mathrm{Ce}$ and Ir are smaller than from other low-abundant contaminants, such as for
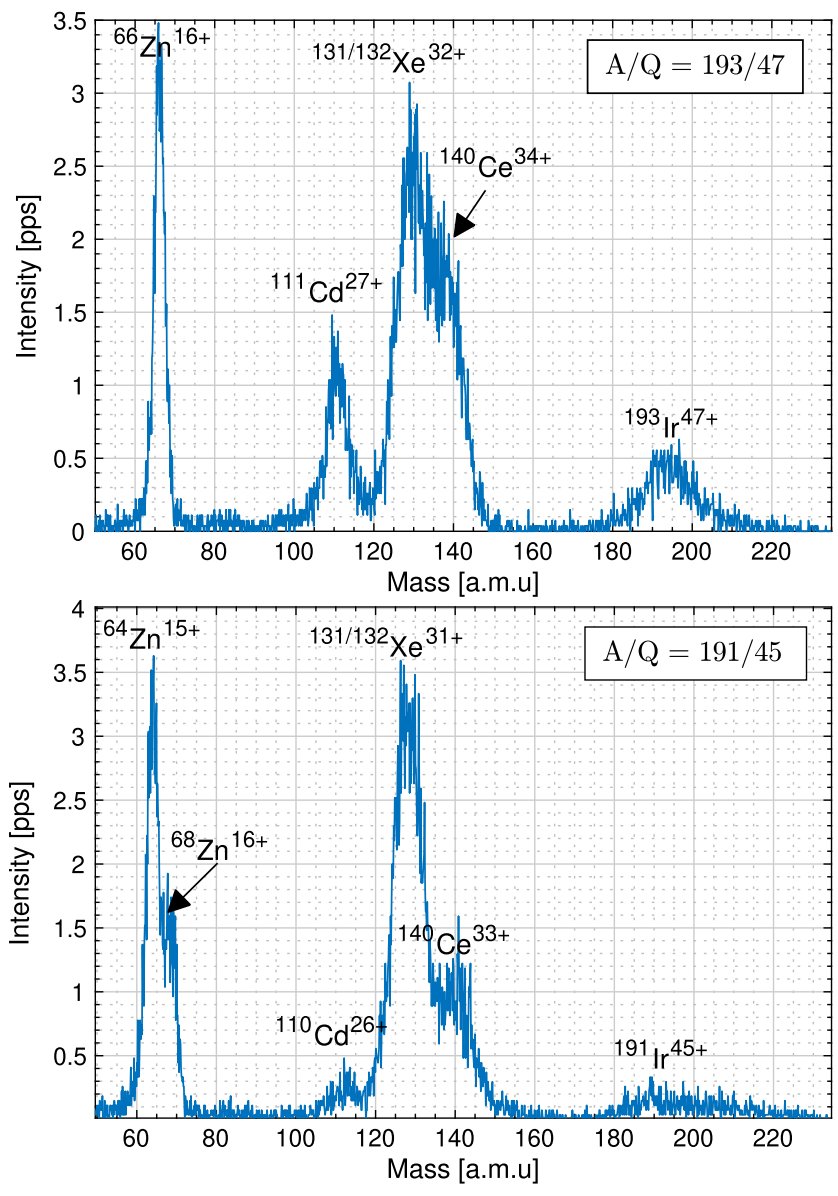

FIG. 27. Particle energy histograms for $A / Q$-selections of 4.21 (top) and 4.244 (bottom) with detected ${ }^{140} \mathrm{Ce}$ and ${ }^{193} \mathrm{Ir}$ peaks together with other contaminants at similar mass-to-charge ratios. 
example Xe isotopes. The absolute counting rate for $\mathrm{Ce}$ is in the order of one particle per second, and even lower for Ir. Hence, we conclude that the beam contamination from the cathode is expected to be negligible for these operational conditions.

\section{CONCLUSION AND OUTLOOK}

In this paper we have presented the design for an electron gun with a target beam current of $500 \mathrm{~mA}$ extracted from a cathode with $2 \mathrm{~mm}$ diameter. As demonstrated with the help of tracking simulations, this immersed gun can be installed in a fringe field of just $700 \mathrm{Gs}$, thanks to the use of a nonadiabatic magnetic field modulation which dampens radial oscillations stemming from the large space charge forces inside the gun volume. Installed at the REXEBIS charge breeder, the electron gun exhibits a perveance of $0.87 \mu \mathrm{A} / \mathrm{V}^{3 / 2}$, which slightly exceeds the simulated value. Due to emission issues with the $\mathrm{IrCe}$ cathode requiring excessive heating, the emission current is currently practically limited to $300 \mathrm{~mA}$, while currents as high as $420 \mathrm{~mA}$ have been punctually achieved.

A crucial requirement for the new electron gun was to maintain the high trapping and breeding efficiency of the REXEBIS setup. Despite the need to reduce the electron beam current, and thereby operating with a lower nominal acceptance than with the original REXEBIS electron gun, we have demonstrated an excellent efficiency and survival rate of the injected ions independent of the confinement time. The presented results, combined with earlier studies, suggest that the emittance of the injected beam can be larger than the calculated acceptance of the electron beam in pulsed injection mode, while still capturing a large fraction of the injected ions. Also, high single charge state yields were demonstrated for long breeding times with ${ }^{205} \mathrm{Tl}$, where the charge state approached the ionization limit imposed by the electron beam energy.

Broad range $A / Q$-spectra have been used to measure the rate of ions created from residual gas in the trapping region. The partial pressures of various gases have been calculated with the help of these scans and provide an estimate of $6.8 \times 10^{-11}$ mbar for the total pressure in the trapping region of REXEBIS. Moreover, measurements have been performed to evaluate the degree of contamination from various isotopes and elements that could evaporate from the new cathode. These were confirmed to be very low, in the range of a particle per second.

Our attempts to measure the current density of the electron beam through charge breeding experiments have revealed a large range of effective current densities depending on the operational parameters. For this purpose we have presented a method of fitting synthetic charge state evolution models to the measured data. We observe a clear trend of higher effective current densities for heavier elements, which agrees with the common notion of improved radial trapping for heavy elements. Due to the large range of reconstructed values it is difficult to assign a single value for the current density of the beam, but the fitting results show some clustering corresponding to a beam radius of approx. $(170 \pm 20) \mu \mathrm{m}$. Since we observe current densities that significantly exceed the theoretical expectation, we assume that the electron beam has a nonuniform current density profile in reality, such that the current density experienced by the ions strongly depends on their radial spread. Furthermore, some of our recorded data-for instance the evolution of ${ }^{39} \mathrm{~K}$ ionsclearly illustrates shortfalls of the Lotz model used for computing the ionization cross sections. It can not accurately reproduce the amplitude of certain charge state yields, particularly close to electron shell closures.

Figure 28 contains a summary of the charge state evolution for various elements from our measurement campaign. It appears that heavy elements in particular benefit from the increased current density, compared to the original REXEBIS setup. This addresses the requests for faster charge breeding of high- $Z$ elements where a low repetition rate has hampered the experiments at the REX/HIE-ISOLDE facility. The first radioactive beam to be charge bred since the upgrade was ${ }^{30} \mathrm{Mg}^{11+}$, where a breeding time of $80 \mathrm{~ms}$ was applied for a $200 \mathrm{~mA}$ electron beam current.

To find supporting evidence for the dynamics of the current density we have performed a number of experiments concerning the evolution of the axial energy distribution of the ions trapped in REXEBIS. The recorded spectra showed a good agreement with Maxwell-Boltzmann distributions with three internal degrees of freedom. While the increasing trend of the fitted ion temperatures and the implied increasing

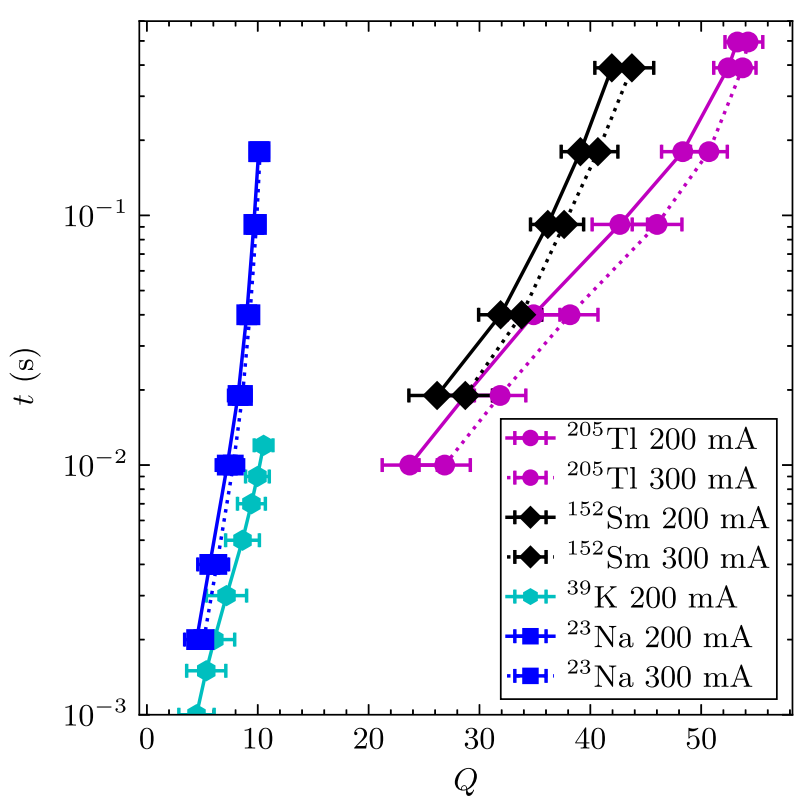

FIG. 28. Plot of the evolution of the mean and standard deviation (error bar) of the charge state distribution of various elements for electron beam currents of $200 \mathrm{~mA}$ and $300 \mathrm{~mA}$. 
radial extent of the ion cloud is in qualitative agreement with the decreasing trend of the effective current density, we were unable to find a mechanism that links the two measurements on a more solid quantitative basis. However, our experiments showed some evidence of effects like heat transfer between light and heavy ions, and a slow dissipation of ion injection energy. The observed heating rates are also in approximate agreement with theoretical values obtained from the Spitzer formula. Based on these observations, it appears interesting to link axial energy measurements to other means of determining the ion cloud characteristics inside the trap, for example through simultaneous recording of $\mathrm{x}$-ray spectra in an electron beam ion trap. REXEBIS does not currently offer the technical means for such a measurement and the solenoid magnet makes it inherently difficult to gain visual access to the trap.

To attack the limited electron emission of the $\mathrm{IrCe}$ cathode, we intend to install and test a novel scandiumdoped dispenser-type cathode developed at Beijing University of Technology, which has previously demonstrated sustained operation at high emission current densities [22]. Work on a modified cathode holder that can be integrated into the existing gun is currently underway and a dedicated test stand for cathode heating experiments is under construction.

Furthermore, our simulation results concerning the reduction of the magnetic field on the cathode show the expected scaling behavior for the current density while the ripple amplitude of the compressed beam is maintained at a low level. An increased beam size is only noted on the first radial excursion prior to traversing the nonadiabatic field modulation. These results suggest that there is room for future performance improvements, with limited modifications to the current design.

\section{ACKNOWLEDGMENTS}

The authors would like to acknowledge the BE-OP-ISO team for assistance with operation of ISOLDE and REX, Miguel Lozano Benito for 3D modelling of the electron gun assembly, and the technical support groups at CERN for production assistance and modifications of the REXEBIS infrastructure, RGA test, metrology measurements and SEM investigations. We acknowledge the contribution of the Research Foundation-Flanders, through the Big Science Project No. G0C2813N. This work has been sponsored by the Wolfgang Gentner Programme of the German Federal Ministry of Education and Research (Grant No. 05E15CHA).Bundesministerium für Bildung und Forschung

[1] F. Wenander, B. Jonson, L. Liljeby, and G. H. Nyman, REXEBIS the Electron Beam Ion Source for the
REX-ISOLDE project, Technical Report No. CERNOPEN-2000-320, CERN, Geneva, 1998.

[2] F. Wenander, J. Cederkäll, B. Jonson, L. Liljeby, G. Nyman, K.-G. Rensfelt, Ö. Skeppstedt, and B. Wolf, REXEBIS, design and initial commissioning results, AIP Conf. Proc. No. 572, 59 (2001).

[3] D. Habs et al. (R.-I. Collaboration), The REX-ISOLDE project, Hyperfine Interact. 129, 43 (2000).

[4] F. Ames, J. Cederkäll, T. Sieber, and F. J. C. Wenander, The REX-ISOLDE Facility: Design and Commissioning Report, CERN Yellow Reports: Monographs (CERN, Geneva, 2005).

[5] B. Wolf, J. Cederkäll, O. Forstner, F. Wenander, F. Ames, K. Reisinger, L. Liljeby, Ö. Skeppstedt, B. Jonson, and G. Nyman, First radioactive ions charge bred in REXEBIS at the REX-ISOLDE accelerator, Nucl. Instrum. Methods Phys. Res., Sect. B 204, 428 (2003).

[6] F. Wenander, P. Delahaye, R. Scrivens, and R. Savreux, The REX-ISOLDE charge breeder as an operational machine, Rev. Sci. Instrum. 77, 03B104 (2006).

[7] F. Wenander, Charge state breeders: On-line results, Nucl. Instrum. Methods Phys. Res., Sect. B 266, 4346 (2008).

[8] F. J. C. Wenander and K. Riisager, Isotope toolbox turns 10, CERN Courier 52, 33 (2012), https://cds.cern.ch/ record/1734740.

[9] N. Warr et al., The Miniball spectrometer, Eur. Phys. J. A 49, 40 (2013).

[10] N. Bidault, J. A. Rodriguez, M. Lozano, and S. Sadovich, Slow extraction of charged ion pulses from the REXEBIS, AIP Conf. Proc. 2011, 070009 (2018).

[11] R. Mertzig, M. Breitenfeldt, S. Mathot, J. Pitters, A. Shornikov, and F. Wenander, A high-compression electron gun for $\mathrm{C}^{6+}$ production: concept, simulations and mechanical design, Nucl. Instrum. Methods Phys. Res., Sect. A 859, 102 (2017).

[12] M. Breitenfeldt, F. di Lorenzo, A. Pikin, J. Pitters, and F. Wenander, MEDeGUN commissioning results, AIP Conf. Proc. 2011, 040004 (2018).

[13] A. Pikin, H. Pahl, and F. Wenander, preceding paper, Method of controlling the cyclotron motion of electron beams with a nonadiabatic magnetic field, Phys. Rev. Accel. Beams 23, 103502 (2020).

[14] F. Wenander, EBIS as charge breeder for radioactive ion beam accelerators, Nucl. Phys. A701, 528 (2002).

[15] Field Precision LLC, Trak Charged Particle Toolkit 8.0 (2017).

[16] Dassault Systemes Deutschland GmbH, CST Studio Suite 2020 SP7 (2020).

[17] G. Herrmann, Optical theory of thermal velocity effects in cylindrical electron beams, J. Appl. Phys. 29, 127 (1958).

[18] G. I. Kuznetsov, IrCe cathodes for EBIS, J. Phys. Conf. Ser. 2, 35 (2004).

[19] R. Mertzig, Modelling and design of high compression electron guns for EBIS/T charge breeders, Ph.D. thesis, TU Dresden, Germany, 2016.

[20] ISTOK, Moscow Russia (private communication).

[21] G. Kuznetsov, High temperature cathodes for high current density, Nucl. Instrum. Methods Phys. Res., Sect. A 340, 204 (1994). 
[22] W. Liang, Y. Wang, J. Wang, W. Liu, and F. Yang, DC emission characteristic of nanosized-scandia-doped impregnated dispenser cathodes, IEEE Trans. Electron Devices 61, 1749 (2014).

[23] M. Breitenfeldt, R. Mertzig, J. Pitters, A. Shornikov, and F. Wenander, The TwinEBIS setup: Machine description, Nucl. Instrum. Methods Phys. Res., Sect. A 856, 139 (2017).

[24] F. Wenander, Charge breeding of radioactive ions with EBIS and EBIT, J. Instrum. 5, C10004 (2010).

[25] F. Ames, G. Bollen, P. Delahaye, O. Forstner, G. Huber, O. Kester, K. Reisinger, and P. Schmidt, Cooling of radioactive ions with the Penning trap REXTRAP, Nucl. Instrum. Methods Phys. Res., Sect. A 538, 17 (2005).

[26] J. Pitters, M. Breitenfeldt, S. D. Pinto, H. Pahl, A. Pikin, A. Shornikov, and F. Wenander, Pepperpot emittance measurements of ion beams from an electron beam ion source, Nucl. Instrum. Methods Phys. Res., Sect. A 922, 28 (2019).

[27] R. Rao, O. Kester, T. Sieber, D. Habs, and K. Rudolph, Beam optics design of the REX-ISOLDE-separator, Nucl. Instrum. Methods Phys. Res., Sect. A 427, 170 (1999).

[28] A. G. Dezfuli, R. Moore, and P. Varfalvy, A compact $65 \mathrm{keV}$ stable ion gun for radioactive beam experiments, Nucl. Instrum. Methods Phys. Res., Sect. A 368, 611 (1996).

[29] E. Kugler, The ISOLDE facility, Hyperfine Interactions 2000 129:1 129, 23 (2000).

[30] T. A. Carlson, C. Nestor, N. Wasserman, and J. Mcdowell, Calculated ionization potentials for multiply charged ions, At. Data Nucl. Data Tables 2, 63 (1970).

[31] I. Podadera-Aliseda, New developments on preparation of cooled and bunched Radioactive Ion beams at ISOL facilities: the ISCOOL project and the rotating wall cooling, Ph.D. thesis, Universitat Politècnica de Catalunya, Barcelona, Spain, 2006.

[32] L. Spitzer and R. Härm, Transport phenomena in a completely ionized gas, Phys. Rev. 89, 977 (1953).

[33] L. Spitzer, Physics of fully ionized gases: Second revised edition, in Physics of Fully Ionized Gases: Second Revised
Edition (Dover Publications, New York, United States, 2006).

[34] F. Currell and G. Fussmann, Physics of electron beam ion traps and sources, IEEE Trans. Plasma Sci. 33, 1763 (2005).

[35] B. M. Penetrante, J. N. Bardsley, D. DeWitt, M. Clark, and D. Schneider, Evolution of ion-charge-state distributions in an electron-beam ion trap, Phys. Rev. A 43, 4861 (1991).

[36] W. Lotz, An empirical formula for the electron-impact ionization cross-section, Z. Phys. 206, 205 (1967).

[37] W. Lotz, Electron-impact ionization cross-sections and ionization rate coefficients for atoms and ions from hydrogen to calcium, Z. Phys. 216, 241 (1968).

[38] W. Lotz, Electron-impact ionization cross-sections and ionization rate coefficients for atoms and ions from scandium to zinc, Z. Phys. A 220, 466 (1969).

[39] Y. S. Kim and R. H. Pratt, Direct radiative recombination of electrons with atomic ions: Cross sections and rate coefficients, Phys. Rev. A 27, 2913 (1983).

[40] R. Mertzig, Project Ion Potentials (2015), http:// project-ionpotentials.web.cern.ch/.

[41] A. Müller and E. Salzborn, Scaling of cross sections for multiple electron transfer to highly charged ions colliding with atoms and molecules, Phys. Lett. A 62, 391 (1977).

[42] J. Angot, M. Luntinen, T. Kalvas, H. Koivisto, R. Kronholm, L. Maunoury, O. Tarvainen, T. Thuillier, and V. Toivanen, Method for estimating charge breeder ECR ion source plasma parameters with short pulse $1+$ injection of metal ions, Plasma Sources Science and Technology 30, 035018 (2020).

[43] R. V. Hogg, A. T. Craig, and J. W. McKean, Introduction to Mathematical Statistics, 8th ed. (Pearson Higher Education \& Professional Group, New York, 2019).

[44] X. Lu and F. J. Currell, Numerical simulation of the charge balance and temperature evolution in an electron beam ion trap, Phys. Rev. ST Accel. Beams 12, 014401 (2009).

[45] N. Bidault, M. Lozano, and J. Rodriguez, Residual gas ions characterization from the REXEBIS, in Proc. 9th International Particle Accelerator Conference (IPAC'18) (JACoW, Geneva, 2018), p. 784, 10.18429/JACOWIPAC2018-TUPAF041. 